\title{
Disziplinäre Nichtkonsolidierung
}

\section{Zu den Anfängen der Mittelalterarchäologie in den 1920er und 1930er Jahren}

Fabian Link

Disciplinary Non-Consolidation. On the Origins of Medieval Archaeology in the 1920s and the 1930s

\begin{abstract}
This article investigates the roots of the sub-discipline medieval archaeology that emerged in German-speaking universities in the 1950s and 1960s. In the 1930s, research practices crucial for the formation of medieval archaeology, such as the investigation of medieval castles and peasant houses, became more prominent in the humanities, especially in the context of völkisch research. After the Nazis took power in Germany, they encouraged such research because it built a scientific basis for their nationalist policy. This politically motivated funding did not result in a new discipline, in contrast to research fields such as prehistory and folklore studies. In this article, I propose two explanations for why medieval archaeology did not emerge as an interdisciplinary research field in the 1930s and 1940s, even though the course was set for its development. First, for archaeologists, art historians, and regional medieval historians, research objects such as medieval castles were semantically too indeterminate. Archaeologists would investigate a castle as a building completely destroyed and buried under rubble, while art historians would be interested in its building technique, and regional medieval historians in its written record. Second, disciplines that were important for the creation of medieval archaeology, such as prehistoric archaeology, art history, and regional medieval history, structurally did not allow for the emergence of an interdisciplinary research field in the 1930s. In particular, prehistoric archaeology, which was crucial for the development of medieval archaeology, itself was not fully institutionalized at universities in the 1930s. This institutionalization process prevented the emergence and development of an interdisciplinary research field such as medieval archaeology. To demonstrate this argument, I draw on two examples of investigations of castles, one in Nazi Germany and the other in the German-speaking part of Switzerland.
\end{abstract}

Keywords: Medieval archaeology, interdisciplinary research practices, formation of disciplines, sociology of science

Schlüsse/wörter: Mittelalterarchäologie, interdisziplinäre Forschungspraktiken, Disziplinenbildung, Wissenschaftssoziologie

In den 1950er und 1960er Jahren etablierte sich die archäologische Subdisziplin Mittelalterarchäologie an den europäischen Universitäten (Fehring 2000; Steuer 1997/1998: 20 f.). In England gründeten Mittelalterarchäologen 1952 die Deserted Medieval Village Research Group, die mittelalterliche Dorfstrukturen und Wüstungen erforschte, und 1957 rief die Society for Medieval Archaeology die erste Zeitschrift für die Archäologie des Mittelalters ins 
Leben. In den 1960er und 1970er Jahren erfolgten weitere Gründungen von Fachzeitschriften und Fachgesellschaften in Italien, Deutschland, der damaligen Tschechoslowakei, Österreich und der Schweiz (Steuer 1997/1998: 24 f.). Charakteristisch für die Mittelalterarchäologie ist ihre interdisziplinäre Praxis, ${ }^{1}$ die aus Methoden, Instrumenten und Konzepten der vor- und frühgeschichtlichen Archäologie, der Kunstgeschichte, der mittelalterlichen Landesgeschichte, am Rande auch der Volkskunde und der Kulturgeografie besteht. Ein weiteres Merkmal dieser archäologischen Subdisziplin ist, dass auch Forschungsbereiche, die zwischen akademischer Wissenschaft und außerakademischer Heimatforschung stehen, für die Mittelalterarchäologie eine wichtige Rolle spielen, wie etwa die Burgenforschung (Biller 1998; Biller and Grossmann 2002; Deutsche Burgenvereinigung 1999; Böhme et al. 2004). Interdisziplinäre Praktiken werden in der heutigen Mittelalterarchäologie insbesondere bei der Erforschung von Objekten wie mittelalterlichen Bauernhäusern oder Burgen angewandt. Der vorliegende Aufsatz behandelt die Geschichte dieser interdisziplinären Subdisziplin, deren Anfänge in den 1920er und 1930er Jahren liegen.

Objekte materieller Kultur des Mittelalters wurden im deutschsprachigen Raum seit dem 19. Jahrhundert, vor allem aber nach dem Ersten Weltkrieg intensiver erforscht. Im Zuge der Nationenbildung im 19. Jahrhundert erwachte ein neues Interesse am Mittelalter, das in den Dienst der historischen Legitimation nationaler und nationalistischer Politik gestellt wurde. Burgen und Dome repräsentierten ein „wehrhaftes Mittelalter“ und symbolisierten eine als genuin deutsch-germanisch imaginierte historische Epoche, die das Römische Reich ablöste. Bereits 1817 gingen Denkmalpfleger, Kunsthistoriker und Politiker daran, die Deutschordensburg Marienburg bei Danzig als Symbol der Überlegenheit deutscher Kultur in Westpreußen zu restaurieren (Falser 2008: 21-34, 39-42). Nach der Reichsgründung 1871 intensivierte Kaiser Wilhelm I. diese Burgenpolitik, die von Wilhelm II. weitergeführt wurde. Die Hohkönigsburg (Haut-Kœnigsbourg) im Elsass, die auf Initiative des Kaisers von 1900-1908 von Burgenforscher und Burgendenkmalpfleger Bodo Ebhardt erforscht und wieder aufgebaut wurde, repräsentierte den deutschen Anspruch auf das Reichsland Elsass-Lothringen (Castellani Zahir 1997). Die Hohenzollernkaiser beabsichtigten mit dieser Kulturpolitik, ihr eigenes Geschlecht in eine Genealogie mit den Stauferkaisern zu stellen, die ihnen als Gründer eines mittelalterlichen germanischen Reichs in Europa galten und die wilhelminische Expansionspolitik legitimieren sollten (DolffBonekämper 2004: 236). Das Mittelalter und seine visuellen Zeugnisse wurden im Laufe des 19. Jahrhunderts zu einem spezifischen Deutungsmuster deutsch-germanischer Kultur, die das Deutsche Reich historisch fundierte (Oexle 2004: 12 f.).

Nach dem Ersten Weltkrieg hielt diese Begeisterung für das Mittelalter in Deutschland an und wurde in der von vielen Deutschen als Krisenzeit 
empfundenen Weimarer Republik aktualisiert. Nationalistische Feiern, bei denen mittelalterliche Symboliken im Zentrum standen, waren nach 1918 ausgesprochen populär. Die „Tausendjahrfeier“ des Rheinlands in Koblenz am 20. Juni 1925 steht exemplarisch für eine Sehnsucht breiter Bevölkerungsschichten nach einer neuen „organischen Ordnung" und der Erwartung eines anbrechenden Reichs nach mittelalterlichem Vorbild. ${ }^{2}$ Für viele deutsche Geistes- und Kulturwissenschaftler stellte das Mittelalter einen utopischen Gesellschaftsentwurf einer besseren Welt dar. Maßgebend hierfür waren die Kriegsniederlage Deutschlands, der Territorialverlust infolge der Versailler Verträge, aber auch eine tiefe intellektuelle Krise, die um 1900 eingesetzt hatte und mit dem Ende des Ersten Weltkriegs ihren Höhepunkt erreichte. 1922 schrieb Paul Ludwig Landsberg in Die Welt des Mittelalters und wir, diese Epoche stelle eine „menschliche Grund- und Wesensmöglichkeit“ dar. Diese lebensphilosophische Interpretation einer historischen Epoche war Ausdruck einer sehnsüchtig erwarteten besseren Sozialordnung, die sich aus den „positiven Kräften“ des Mittelalters konstituieren sollte (Oexle 1996: 139_ 142). Angehörige der „konservativen Revolution“ vertraten solche Denkfiguren besonders prominent. In diesem sozialen Milieu wurde nach 1918 ein „Hunger nach Ganzheit“ mit Schwärmereien von einem kommenden Reich und einem territorial- und bevölkerungspolitischen Revisionismus amalgamiert, den jungkonservative Intellektuelle der „Entzauberung der Welt“ (Max Weber) entgegenhielten (Oexle 1996: 142-144). Im wissenschaftlichen Feld war dieses ideologische und kulturpolitische Aggregat richtungsweisend für den hauptsächlich aus jungkonservativen und revisionistisch gesinnten Kulturwissenschaftlern bestehenden Forschungsverbund der „Volksdeutschen Forschungsgemeinschaften" (VFG), ein 1931 gegründetes informelles Netzwerk, das sich der Erforschung des „deutschen Westens, Ostens und Südostens" verschrieben hatte (Fahlbusch 1999: 65-73, 2000). Die Erforschung des mittelalterlichen Reichs und mittelalterlicher Kultur nahm in diesem Forschungsverbund eine herausragende Stellung ein.

Auch außerhalb Deutschlands, vor allem in der deutschsprachigen Schweiz und in Österreich, erforschten Wissenschaftler in Zusammenarbeit mit historischen Laiengesellschaften seit den 1920er Jahren vermehrt mittelalterliche Siedlungen und Burgen (vgl. Bentz 2008). In allen deutschsprachigen Regionen Europas war die seit dem 19. Jahrhundert gesteigerte Popularität mittelalterlicher Monumente mit einer nationalen bis völkisch-tribalistischen Erinnerungskultur verbunden. So waren Burgen für die Bündische Jugend Symbole ihres romantisch-utopischen Gesellschaftsentwurfs (Fleischner 1999: 39-41), und die internationale Arbeitsdienstbewegung, die als Reaktion auf die ökonomischen, politischen und kulturellen Krisen in der ersten Hälfte des 20. Jahrhunderts entstanden war, setzte junge Arbeitslose bei Ausgrabungen und Restaurierungen von Burgen ein, um den Jugendlichen eine nationale und heimatbezogene Haltung nahe zu bringen (vgl. Patel 2003). ${ }^{3}$ 
Im NS-Regime hatten mittelalterliche Monumente eine besondere Relevanz, da NS-Ideologen mit Burgen, Schlössern und altdeutschen Bauernhäusern das „Dritte Reich“ historisch zu legitimieren suchten (vgl. Pütz 2002; Siebert 1941: 9; Teibenbacher 2003). Auch wenn es kein einheitliches nationalsozialistisches Mittelalterbild gab, waren sich NS-Politiker und NSIdeologen doch darin einig, dass das Mittelalter eine zentrale historische Epoche für die Legitimation und Demonstration der Überlegenheit deutscher Kultur und Politik darstellte (Wolnik 2004: 252). Zudem bildete die Vorstellung von einer „organischen“ Sozialordnung im Mittelalter, bestehend aus dem Kaiser oder „Führer“ und seinem hierarchisch geordneten Gefolge, die Idealvorstellung für den zeitgenössischen „Führerstaat“ (Schreiner 1985: 175). Burgen waren dabei Objekte besonderer Faszination. Es verwundert daher nicht, dass Vertreter der NS-Elite der Burgendenkmalpflege und der Burgenforschung verstärkte finanzielle Unterstützung zukommen ließen. Adolf Hitler förderte mehrere Wiederaufbauten und Restaurierungen von mittelalterlichen Burgen mit namhaften Beiträgen, so die Ruine Wildenberg im Odenwald, die Burg Lauenstein oder das niedergebrannte Stuttgarter Schloss. ${ }^{4}$ Darüber hinaus versprach er 800.000 RM für die Renovierung der Burgen des Deutschen Ordens in Osteuropa ${ }^{5}$ und spendete 400.000 RM für ein Wiederaufbau- und Konservierungsprogramm von Schlössern und Burgruinen in Bayern und der Pfalz (Backes 1988: 185). Mit diesen Unternehmen der NSKulturpolitik waren in vielen Fällen wissenschaftliche Untersuchungen verbunden, die entweder aufgrund der Einwilligung des jeweiligen NS-Politikers zustande kamen oder deshalb durchgeführt wurden, weil ein Ausgrabungsund Denkmalschutzgesetz verlangte, dass vor einer Restaurierung der historische Bestand wissenschaftlich zu dokumentieren sei. Einzelne NS-Ämter und NS-Politiker interessierten sich genuin für die Erforschung des Mittelalters. So existierte in den 1930er Jahren in Heinrich Himmlers Forschungsverbund, der „Forschungsgemeinschaft Deutsches Ahnenerbe e.V.“, eine „Forschungsstätte für germanisches Bauwesen“, deren Leiter Martin Rudolph spätmittelalterliche und frühneuzeitliche Bauernhäuser untersuchte. $^{6}$

Die Beschäftigung von Archäologen, Landes- und Kunsthistorikern sowie Volkskundlern mit Burgen, Schlössern und mittelalterlichen Bauernhäusern in den 1920er und 1930er Jahren war verbunden mit einer ideologischen und politischen Verwendbarkeit dieser Objekte für NS-Politiker und NS-Ideologen oder für heimatlich oder national gesinnte Kulturpolitiker in Ländern wie der Schweiz, eine Tradition, die bis ins 19. Jahrhundert zurückging.

Die vermehrte Erforschung mittelalterlicher Objekte seit den 1920er Jahren und die im NS-Regime intensivierte Förderung von Ausgrabungen und Restaurierungen von mittelalterlichen Objekten geben Anlass zur Vermutung, dass spätestens in den 1930er Jahren ein Forschungsfeld entstanden war, das den Boden bereitet hatte für die Mittelalterarchäologie der 1950er und 1960er 
Jahre. Ausgang dieser Hypothese ist die Annahme, dass die an diesen Feldforschungsunternehmen beteiligten Wissenschaftler eine Zusammenarbeit untereinander anstrebten, um ihre Forschungsergebnisse zu optimieren und ihr Forschungsfeld institutionell zu verankern. Die Annahme, dass die Mittelalterarchäologie sich bereits in den 1930er Jahren zu einem disziplinären Gebilde verdichtet hatte, weist mehrfache Evidenz in den Quellen auf. Bolko von Richthofen, ein Vor- und Frühhistoriker, der seine Wissenschaft völkischrevisionistischen Zielsetzungen in Bezug auf die ehemals deutschen Ostgebiete unterstellte, hatte in den späten 1920er Jahren eine Vorstellung von einer „Archäologie des Mittelalters“ entwickelt, die dem heutigen Verständnis von Mittelalterarchäologie nicht ganz unähnlich ist. Für Richthofen war die archäologische Erforschung des Mittealtelters ein Forschungskonzept, bei dem „Prähistoriker, Historiker und Kunsthistoriker [...] durch die Ausbildung der mittelalterlichen Archäologie gefördert" werden müssten und „gemeinschaftlich" zusammenarbeiten sollten. ${ }^{7}$ Zentral in Richthofens programmatischem Vorschlag war das zeitgenössische Konzept der "Gemeinschaftsforschung“. Mit Gemeinschaftsforschung oder „Forschungs-" und "Arbeitsgemeinschaft" war eine institutionalisierte oder nur lose Organisation von Wissenschaftlern verschiedener Disziplinen gemeint, die zu einer übergreifenden Fragestellung arbeitete (vgl. Hachtmann 2007; Hausmann 2001; Szöllösi-Janze 2000). In den 1920er und 1930er Jahren existierten viele solcher Arbeitsgemeinschaften, die nach 1933 dann in „Reichsarbeitsgemeinschaften“ unbenannt wurden, wie beispielsweise die „Reichsarbeitsgemeinschaft für Volksforschung“ oder die „Reichsarbeitsgemeinschaft für Raumforschung“. Es wäre also zu vermuten, dass die Entstehung der Mittelalterarchäologie im Kontext der Gemeinschaftsforschung der 1920er und 1930er Jahre erfolgte, weil ihre Arbeitsform interdisziplinären Charakter hatte.

Ein Hinweis darauf, dass eine solche Entwicklung insbesondere durch die Fokussierung auf Objekte wie Burgen erfolgte, findet sich in einem 1937 publizierten Aufsatz des Landeshistorikers Hermann Aubin. Aubin zufolge sollte sich die Burgenforschung als ein "Sondergebiet der Siedlungskunde" entwickeln, in der ganze Burgensysteme und die Zusammenhänge mit dem „Vom Burgengürtel umhegten Siedlungsland“ untersucht würden (Aubin 1937: 571). In der Zeitschrift Deutsches Archiv für Landes- und Volksforschung, die Mitglieder der VFG 1937 gründeten, finden sich zahlreiche Beiträge, in denen sich die Autoren mit der Geschichte der Burgen befassten. ${ }^{8}$ Burgenforschung war offenbar ein Scharnierbereich zwischen Landes- und Kunstgeschichte, Archäologie und Siedlungsforschung. An der Burgenforschung müsste sich demnach die Verschiebung von einem disziplinär gebundenen methodischen Herangehen zu einem problem- und objektorientierten Ansatz zeigen, die der Herausbildung des interdisziplinären Forschungsfelds Mittelalterarchäologie Vorschub geleistet hätte. 
Solche disziplinären Verschiebungen und Verdichtungen sind auch in anderen geistes- und kulturwissenschaftlichen Bereichen festgestellt worden. Die offenbar wachsende Zusammenarbeit von Wissenschaftlern unterschiedlicher Disziplinen und Fächer seit den 1920er Jahren, an welcher insbesondere Forscher jungkonservativer politisch-ideologischer Prägung beteiligt waren, hat Willi Oberkrome $(1991 ; 1993)$ und andere Autorinnen und Autoren zur Interpretation veranlasst, dass eine revisionistische und von völkischen Ideologemen unterlegte Strömung in der deutschen Geschichtswissenschaft nach dem Ende des Ersten Weltkriegs eine neue interdisziplinäre Forschungsrichtung begründet habe, nämlich die "Volksgeschichte“ oder „Volksforschung“. Durch vielfältige Umwandlungen habe dieses interdisziplinäre Forschungsfeld dann die methodologische Basis der westdeutschen Struktur- und Sozialgeschichte der 1950er und 1960er Jahre dargestellt. Hierbei spielten die VFG eine besonders wichtige Rolle. Die Mitglieder dieses wissenschaftlichen Netzwerks operierten nicht mehr entlang der herkömmlichen Fächer- und Disziplinengrenzen, sondern richteten ihre Fragestellungen nach völkischen Gesichtspunkten und politischen Zielsetzungen aus, wie beispielsweise der wissenschaftliche Nachweis, dass die Regionen Elsass und Lothringen „volksdeutscher Kulturboden“ seien. Auch in der Volkskunde sind ähnliche Entwicklungen zu beobachten. Am Beispiel von volkskundlichen Großunternehmen wie dem Atlas der deutschen Volkskunde hat Friedemann Schmoll (2009: 120) gezeigt, dass die disziplinäre Gebundenheit von Forschungspraktiken in den 1930er Jahren zugunsten einer problemorientierten Herangehensweise substituiert wurde.

Der Ausbau von bestimmten Disziplinen und die Formierung neuer Forschungsfelder im NS-Regime waren eng mit der Verwendbarkeit der jeweiligen Forschungsobjekte und des von den jeweiligen Wissenschaftlern produzierten Wissens für NS-Politiker verbunden. Professionalisierungstendenzen und Etablierungsprozesse von Forschungsbereichen im akademischen Feld aufgrund einer verstärkten Förderung durch Nationalsozialisten sind für verschiedene Fächer beobachtet worden, angefangen mit der Psychologie, die verwendbares Wissen der Wehrmacht bereitstellen konnte (Geuter 1984). Für Disziplinen und Forschungsbereiche, die für die spätere Mittelalterarchäologie entscheidend waren, gilt dies im besonderen Maße. Nebst der bereits erwähnten Volkskunde sind die vor- und frühgeschichtliche Archäologie (Leube \& Hegewisch 2002), die mittelalterliche Landesgeschichte (Wallraff 2008), die ihrerseits eng mit der „Volksgeschichte“ verbunden war, sowie die mittelalterliche Kunstgeschichte (Heftrig et al. 2008) zu nennen. In der Vorund Frühgeschichte wirkte sich die politische Verwendbarkeit des von Archäologen produzierten Wissens auf die institutionelle Struktur dieses Faches besonders deutlich aus: Gab es in der Vor- und Frühgeschichte 1929 lediglich sechs ordentliche Lehrstühle und außerordentliche Professuren in Deutschland und Österreich, war die Zahl der Professuren in beiden Ländern 
bis 1942 auf 25 angestiegen, von denen 17 ordentliche Lehrstühle waren (Pape 2002: 168). Sowohl für die Vor- und Frühgeschichte als auch für die Landesgeschichte ist hierbei anzumerken, dass dieser institutionelle Ausbau bereits vor der sogenannten Machtergreifung der Nationalsozialisten eingesetzt hatte, jedoch von NS-Wissenschafts- und Kulturpolitikern katalysiert wurde. Aufgrund der oben dargelegten politischen Relevanz von Objekten mittelalterlicher Kultur im NS-Regime wäre demnach ein Anstieg mittelalterlicher kulturwissenschaftlicher Forschung auf interdisziplinärer Basis zu erwarten.

Der vorliegende Aufsatz diskutiert die Anfänge der kulturwissenschaftlichen Mittelalterforschung unter der Annahme, dass durch die Intensivierung interdisziplinärer Praktiken und die gesteigerte Bedeutung kulturwissenschaftlicher Fächer in den 1920er und vor allem in den 1930er Jahren ein neues Forschungsfeld entstanden war, das als Basis für die spätere akademische Subdisziplin Mittelalterarchäologie anzusehen ist. Diesen Prozess möchte ich mit einem wissenschaftssoziologischen und einem praxeologischen Ansatz angehen und anhand zweier Fallbeispiele von Burgenforschung in den 1930er Jahren die Forschungspraktiken der daran beteiligten Akteure untersuchen.

\section{Methodische Standards in der kulturwissenschaftlichen Mittelalterforschung und die Burg als Wissensort und epistemisches Objekt}

Bevor ich zu den beiden Burgenforschungsprojekten übergehe, muss bestimmt werden, welche methodischen und technischen Standards in den frühen 1930er Jahren in den Disziplinen gängig waren, die für die spätere Mittelalterarchäologie ausschlaggebend sein sollten. Zudem soll die Burg als Wissensort und epistemisches Objekt näher definiert werden.

\section{Methodische Standards in den 1920er und 1930er Jahren}

Für die Mittelalterarchäologie der 1950er und 1960er Jahre war entscheidend, dass Archäologen, Kunst- und Landeshistoriker ihre jeweils eigenen Methoden, Praktiken und Konzepte in einen neuen, disziplinenübergreifenden Kanon überführten (vgl. Steuer 1997/1998: 20). Hierfür waren ähnliche methodologische Übersetzungsleistungen ausschlaggebend, wie sie Carsten Reinhardt (2001: 1-13) für die Entstehung der Biochemie in den 1960er und 1970er Jahren beschrieben hat. In der chemischen Forschung dieser Zeit wurden physikalische und biologische Methoden in die Chemie importiert, um die Glaubwürdigkeit der Chemie im Wissenschaftsfeld zu erhöhen, da sich die Chemiker in einer „methodischen Krise“ sahen. „To make instruments chemical - in devising useful methods for chemists - was [...] a strategy of 
chemists for creating disciplinary space" (Reinhardt 2006: 1). Die Mittelalterarchäologie als ein solch neuer „disziplinärer Raum“ entstand allerdings nicht aufgrund eines Krisenmoments einer an ihrer Formierung beteiligten Wissenschaften, sondern weil die Akteure ihre Forschungsobjekte möglichst "total" untersuchen wollten, was nur durch eine Kombination verschiedener Techniken und Methoden geleistet werden konnte. Das entscheidende methodische Prinzip dabei war das aus der Archäologie stammende stratigrafische Vorgehen, also das Abtragen und Dokumentieren der Befunde und Funde nach deren Schichtung. Baustrukturen von Burgen und Bauernhäuser sollten mit dieser Methode genauso untersucht werden wie die Kulturschichten im Boden.

In den 1920er Jahren sind solche methodologischen Transfer- und Übersetzungsleistungen kaum festzustellen. Es liegt nur ein einziges Beispiel eines Forschungsunternehmens vor, bei dem es einem Wissenschaftler gelang, einen einheitlichen Methodenkanon für die Untersuchung von Bodenstrukturen, Mauerwerk und historischen sowie bildlichen Quellen anzuwenden, nämlich die Untersuchung des Schlosses Hallwyl von 1910 bis 1916 durch den schwedischen Archäologen und Volkskundler Nils Lithberg (Motschi 2000: 84 f.). Auf Basis des derzeitigen Forschungsstands muss dieses Unternehmen als eine Ausnahme angesehen werden. Litherg, der in der Wissenschaftlergemeinschaft eine relativ isolierte Position einnahm, entfaltete mit seinen Forschungen keine Wirkung im Sinne einer strukturellen Verschiebung in den kulturwissenschaftlichen Disziplinen. In den 1920er Jahren war es vielmehr üblich, mit den Methoden und Techniken der je eigenen Disziplin, in der die jeweiligen Wissenschaftler sozialisiert worden waren, Burgen, Schlösser oder mittelalterliche Bauernhäuser zu untersuchen. Dazu gehörten die vor- und frühgeschichtliche Archäologie, die Landesgeschichte, die Kunstgeschichte sowie Kulturgeografie und Volkskunde.

Seit dem 19. Jahrhundert ist die Typologie zu den Standardmethoden in der vor- und frühgeschichtlichen Archäologie zu zählen. Hierbei handelt es sich um die quantitative und qualitative Kategorisierung von Fundobjekten nach Material, Funktion und Stil. Abbildung 1 zeigt eine charakteristische Montage von Fundzeichnungen mit dazugehöriger Beschreibung des jeweiligen Artefakts. Auch Versuche, Objekte wie Burgen nach Gesichtspunkten wie Grundriss oder Baustil zu typologisieren und sie im Zusammenhang mit der Umgebung, in der sie gebaut wurden, zu ordnen (Höhenburg, Wasserburg, Höhlenburg usw.), gehörten zu den etablierten methodischen Herangehensweisen (vgl. Schuchhardt 1931). Eine wichtige Technik, die im Laufe der 1920er Jahre zentraler wurde und die auf eine zunehmende Zusammenarbeit zwischen Archäologen und Landeshistorikern hindeutet, war die Kartierung von Fundplätzen und von Funden. Auf diese Weise ließ sich das Vorkommen einer bestimmten Keramikform bei einem Ausgrabungsplatz mit den vorgefundenen Keramikformen bei anderen Ausgrabungsstellen in eine Beziehung 
setzen. Archäologen eruierten aufgrund der räumlichen Darstellung des Auftretens bestimmter Keramikformen (oder anderer Fundkategorien) Siedlungs- und Wanderungsbewegungen ethnisch definierter Gruppen (Abb. 2). Wie Susanne Grunwald (2014) allerdings betont, existierten in den 1920er Jahren kaum verbindliche Standards für die vor- und frühgeschichtliche Kartierungstechnik. Vielmehr arbeitete jeder Forscher und jede Forschergruppe nach eigenem Gutdünken.

Besonders wichtig für die Vor- und Frühgeschichte war die Verwendung von naturwissenschaftlichen Methoden zur Untersuchung der Bodenstrukturen und der sich darin befindlichen Funde. Die naturwissenschaftliche Methodik wurde insbesondere nach dem Ersten Weltkrieg weiterentwickelt. Hierzu sind archäozoologische und archäobotanische Untersuchungen zu zählen, also Analysen von Resten der Flora und Fauna. Innovative Ausgräber wie Gerhard Bersu, Hans Reinerth oder Herbert Jankuhn konzipierten in dieser Zeit auch neue Grabungstechniken. $\mathrm{Zu}$ nennen ist die verbesserte Systematik der Befundaufnahme und Dokumentation, die es erlaubte, Fundobjekte und Befunde innerhalb der jeweiligen Ausgrabungssektoren miteinander in Beziehung zu setzen oder der Einsatz von Wasserpumpen bei Feuchtbodensiedlungen (vgl. Krämer 2002; Schöbel 2002). Abbildung 3 zeigt eine schematische Einteilung eines Ausgrabungsplatzes in Grabungsabschnitte, wobei die archäologischen Befunde innerhalb dieser Grabungssektoren mit Nummern gekennzeichnet wurden. Dieses Nummernsystem durfte während der gesamten Ausgrabung nicht verändert werden, sodass eine eindeutige Zuordnung der jeweiligen Befunde garantiert werden konnte.

Kulturgeografisch ausgerichtete Landeshistoriker bedienten sich vornehmlich schriftlicher Quellen, wie es in historischen Wissenschaften üblich war. Hierbei ist die Erforschung von Rechtsquellen hervorzuheben, die Rückschlüsse auf die mittelalterlichen Herrschaftsstrukturen ermöglichte, wobei Burgen oder Klöstern eine zentrale Funktion zukam (vgl. Merz 19091914; Piper 1912). Diese Methode sollte für die spätere Mittelalterarchäologie eine entscheidende Rolle spielen, weil auf diese Weise materielle Kultur und historische Methoden miteinander verbunden werden konnten: Das im Archiv aufbewahrte Inventar einer Burg, das auf den Zeitpunkt der Erhebung des Inventars datiert, konnte mit dem innerhalb und außerhalb des Burgareals aufgefundenen und mit der stratigrafischen Methode relativchronologisch bestimmten Fundmaterial abgestimmt werden. Eine weitere zentrale Methode in der Landesgeschichte der 1920er Jahre war die Erstellung von historischen Karten eines spezifischen Herrschaftsgebietes. Das der Kulturgeografie entliehene historisch-kartografische Verfahren war eine kondensierte und wirksame Darstellungsform komplexer historischer Zusammenhänge und Prozesse und sollte für die Entwicklung der „Volksgeschichte“ ausschlaggebend sein. Konfessionelle Gliederungen konnten damit genauso dargestellt 


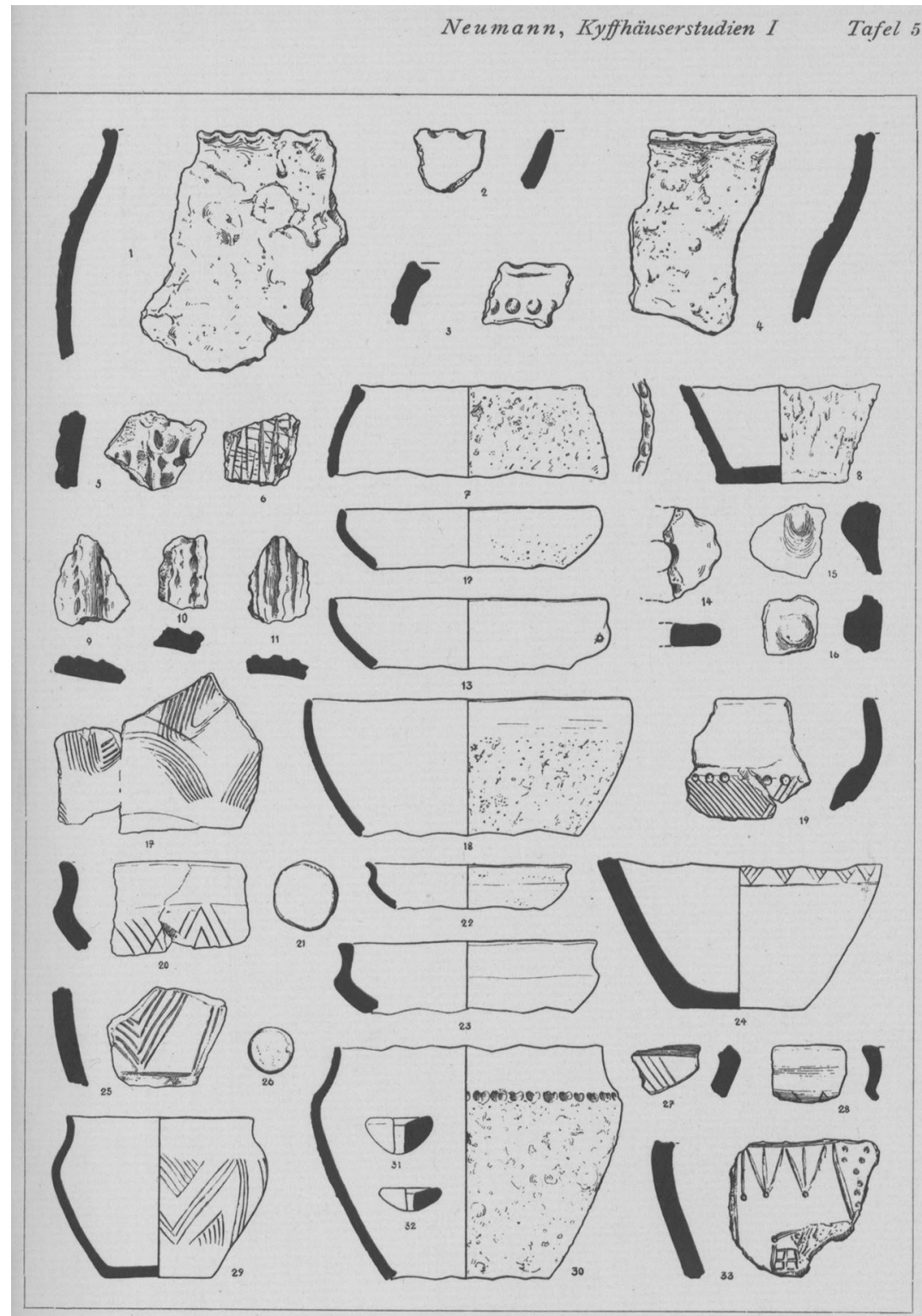

Abb. 2. Kyffhäuser, Oberburg: Vorgeschichtliche Irdenware. I:4.

Zeichn.: G. Keil, R. Kuboschek, Jena

Abb. 1 Ausschnitt aus dem Fundkatalog der Ausgrabung der mittelalterlichen Burgruine auf dem Kyffhäuser Burgberg (1937-1938). Die Abbildung zeigt plastisch gezeichnete Funde, die auf die Tafel montiert wurden. Die Profilzeichnung und die plastische Darstellung der archäologischen Funde sollten Evidenz dafür erzeugen, sie bereits bekannten Fundtypen zuzuordnen (Neumann 1940: Tafel 5) 


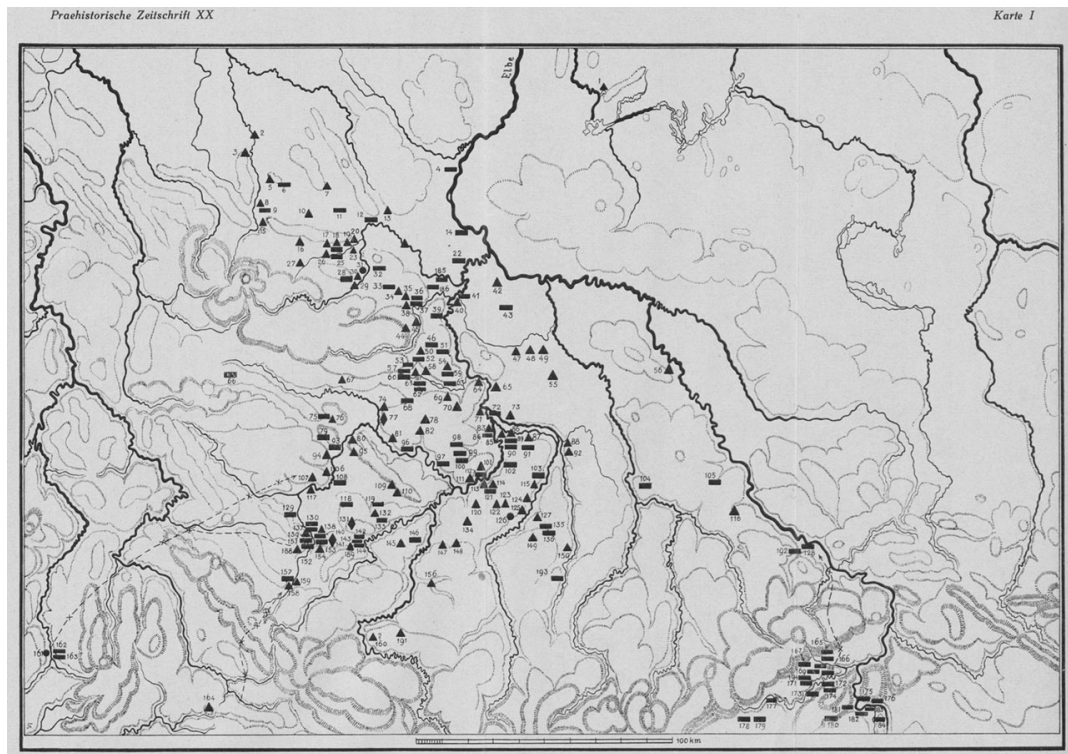

Abb. 2 Karte von Fundstellen von Objekten der "(böhmisch-)sächsischen" und „(hessisch-) thüringischen" Gruppe innerhalb der neolithischen Glockenbecherkultur. Die Kartierung solcher Fundstellen im geografischen Raum erlaubte das Erkennen von strukturellen Zusammenhängen, was in den 1920er und 1930er Jahren mit Wanderungen von ethnisch definierten Gruppen in ein Verhältnis gesetzt wurde (Neumann 1940: 327)

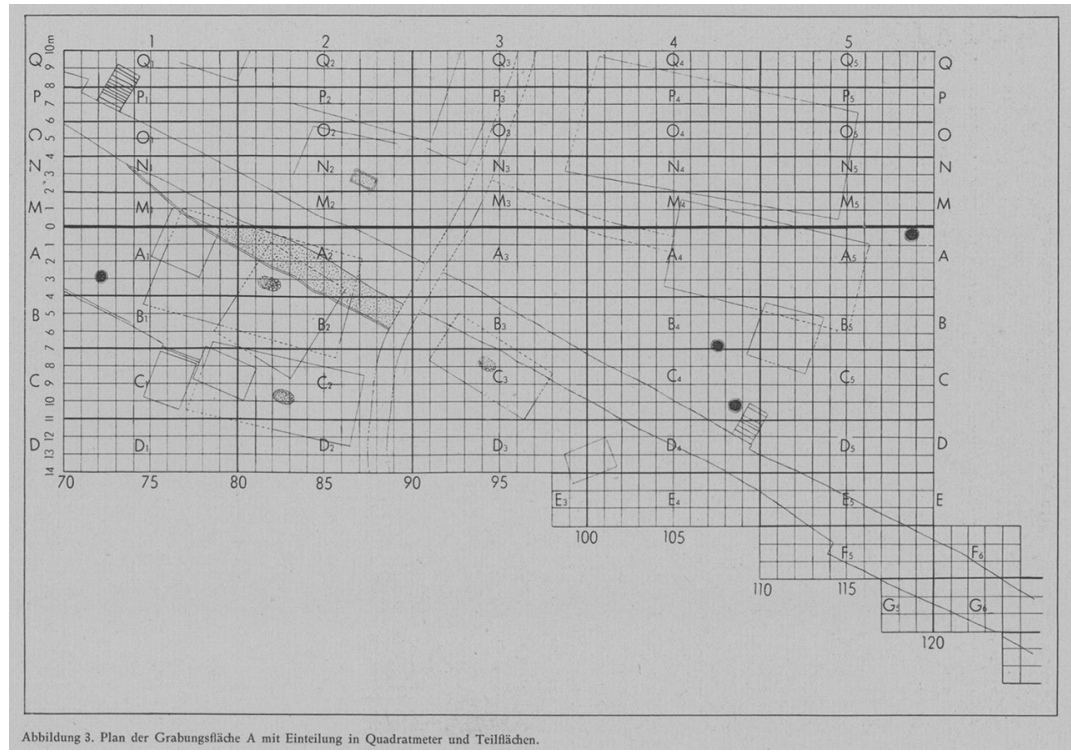

Abb. 3 Aufteilung eines Teilbereichs des Ausgrabungsplatzes von Haithabu, einer wikingerzeitlichen Handelsstadt, in Untersektoren. Die Befunde sind in das Koordinatenraster eingehängt, sodass sie genau lokalisiert werden konnten (Jankuhn 1943: 17, Tafel 4) 
und analysiert werden wie die herrschaftlichen Beziehungen einzelner mittelalterlicher Fürsten zueinander, die anhand der Lage ihrer Burgen im kulturgeografischen Raum aufgeschlüsselt wurden (vgl. Oberkrome 1993: 47, 67).

In der architekturhistorisch ausgerichteten Kunstgeschichte und der Denkmalpflege stellten stilistische Analysen der Bauplastik und des Baumaterials das übliche methodische Vorgehen dar (vgl. Die Denkmalpflege) Deutsche Kunst und Denkmalpflege 1899-1935). Fotografien, meist allerdings mit eher ästhetischer denn analytischer Funktion, gehörten seit dem späten 19. Jahrhundert zu den methodischen Standards in der kunsthistorischen Bauforschung (vgl. Ebhardt 1898-1908). In den 1920er Jahren begannen Bauforscher, naturwissenschaftlich-technische Methoden anzuwenden, wie etwa die Mörtelanalyse, bei der die Zusammensetzung von Mörteln im Labor analysiert wurde. ${ }^{9}$ In Verbindung mit der stratigrafisch-archäologischen Methode konnten dadurch verschiedene, aufgrund einer mehr oder weniger eindeutigen Struktur des Mörtels festgelegte Bauteile eines mittelalterlichen Stadthauses oder einer Burg miteinander in ein Verhältnis gesetzt werden, um den Bauablauf des jeweiligen Gebäudes zu bestimmen.

Diese Auflistung der verschiedenen Methoden, mit denen mittelalterliche Kulturobjekte in den 1920er und frühen 1930er Jahren erforscht wurden, verdeutlicht, dass keine einheitliche methodische Grundlage in denjenigen Wissenschaften existierte, die für die spätere Mittelalterarchäologie ausschlaggebend sein sollten. Nur wenige fächerübergreifende Standards wie die Fotografie oder die Typologie lassen sich festmachen, allerdings wurden auch sie in den einzelnen Disziplinen unterschiedlich angewandt.

\section{Die Burg als Wissensort und epistemisches Objekt}

Eine Burg ist ein Wissensort und ein epistemisches Objekt zugleich. Als Ort der Produktion von Wissen weist sie andere Charakteristiken als das Labor oder das Archiv auf. Im Gegensatz zu diesen "geschlossenen“, einerseits von der Umwelt deutlich abgetrennten, andererseits gesellschaftlich-institutionell von symbolischen Grenzen umgebenen Orten, muss eine Burg zuerst in einen Wissensort und in ein epistemisches Objekt verwandelt werden. Hierzu gehört die Schaffung möglichst klarer Grenzen zwischen wissenschaftlichem und nicht wissenschaftlichem Terrain durch die Installation von wissenschaftlichen Instrumentarien und einer Infrastruktur. Archäologen und Bauforscher müssen ihre Instrumente auf der entsprechenden Burg aufstellen und Lagerplätze für die Grabungswerkzeuge sowie eine Hütte für das Lagern von Plänen, Zeichnungen und Fotografien errichten. Um den Schutt abzuführen, müssen Vorrichtungen gebaut werden, da Steine, Sand und Erde in der Regel nicht einfach den Burgberg hinuntergeworfen werden können. Solche Abgrenzungen des Untersuchungsorts zur natürlichen Umgebung sind für alle Feldwissenschaften charakteristisch. Hierzu gehören auch die stetigen 
Bemühungen der Feldforscher, die teils verheerenden Wirkungen von Umwelteinflüssen (Regen, Schnee, Wind), die außerhalb der menschlichen Kontrolle stehen, durch Unterstände oder Abdeckungen der Ausgrabungsflächen abzuschwächen (Kohler 2002: 6).

Bei den Praktiken der archäologischen und kunsthistorischen Feldarbeit handelt es sich nicht um wiederholbare Abläufe wie in den Laborwissenschaften. Archäologen und in geringerem Masse auch Bauforscher zerstören vielmehr ihre Untersuchungsgegenstände, denn jede Kulturschicht, die untersucht werden soll, muss abgebaut werden. Durch das Abtragen einer Kulturschicht wird ein irreversibler Zustand herbeigeführt; die untersuchte Schicht bleibt zerstört und kann nicht in einem nachfolgenden Arbeitsschritt nochmals erforscht werden (vgl. Eberhardt 2008). Hierbei sind Geschicklichkeit und Beobachtungsgabe gefordert, wofür eine spezifische Art des Sehens und Dokumentierens ausschlaggebend ist. Der archäologische oder kunsthistorische Blick wird in Kollektiven gebildet, so etwa in relativ homogenen Ausgrabungsmannschaften oder Bauforschergruppen, die regelmäßig zusammenarbeiten (vgl. Fleck 1980: 85). Nur durch ein spezifisch gerichtetes Sehen und Hantieren mit Instrumenten, das sich in diesen Gruppen oder Kollektiven etabliert hat, können die Feldforscher kulturhistorische Spuren erkennen, diese nach bestimmten Methoden dokumentieren und sie nach einem im Kollektiv festgelegten Kategorienraster interpretieren (Fleck 1983: 148, 153 f., 157).

Die Feldforscher halten das technische Vorgehen und die getroffenen Beobachtungen auf Medien, wie Foto- oder Millimeterpapier fest. Die Aufzeichnungen werden ,mobil' gemacht, sodass sie vom Untersuchungsort an andere Orte verlagert werden können (beispielsweise in die Büros eines Landesdenkmalamts), ohne dass das darauf festgehaltene Wissen verändert wird. Diese Inskriptionen stellen Repräsentationen von Dingen oder von Zuständen dar, die zu einem bestimmten Zeitpunkt bei der Ausgrabung oder der Bauuntersuchung beobachtbar waren (vgl. Hagner 1997). Viele dieser Zustände werden im Laufe einer Ausgrabung oder Bauuntersuchung auf Millimeterund Fotopapier, in Notizbüchern oder auf losen Skizzenblättern festgehalten und bilden in ihrer Gesamtheit die „optische Konsistenz“ der Dokumentation (Latour 2006: 271, 278). Zweck der Dokumentation ist, den gesamten Ablauf der Untersuchung als konzises Ganzes und als einen Prozess logisch aufeinanderfolgender Arbeitsschritte darzustellen. Hierfür werden Pläne der erhaltenen Mauern hergestellt, auf denen die neu zutage geförderten Mauerreste eingetragen werden, Fundobjekte werden möglichst in dem Befundzusammenhang fotografiert, in dem sie aufgefunden und für die Fotografie präpariert wurden. Die Ausgräber zeichnen Profile, also senkrechte Wände von Ausgrabungsschnitten oder Schächten, bei denen die Abfolge der Kulturschichten deutlich wird, und beschreiben diese Schichten und die darin enthaltenen Befunde und Funde (Abb. 4). Die einzelnen Bestandteile des 
Wissensobjekts Burg werden nach und nach in Millimeter- und Fotopapier, Notiz- und Skizzenbücher transdisponiert.

Einmal auf Papier übertragen, also mobil und unveränderlich zugleich gemacht, können die archäologischen Wissenselemente in ihrem Maßstab modifiziert, reproduziert, neu kombiniert und interpretiert werden. Der chronologische Ablauf von Kulturschichten und Bauteilen ergibt sich in der Regel erst durch das Zusammenhängen und Vergleichen verschiedener Dokumentationsstufen. Im günstigen Fall werden zum Schluss die Inskriptionen in einen Text verwandelt, denn wie in anderen Wissenschaften ist auch in der Archäologie und der kunsthistorischen Bauforschung der Text das zentrale Mittel zur Vermittlung von Plausibilität des generierten Wissens an die Fachgemeinschaft und die Öffentlichkeit (Latour 2006: 276, 285-287). Die Praxis des Inskribierens sollte dabei nach einheitlichen Kriterien erfolgt sein, um eine möglichst hohe Plausibilität des Wissens zu erzeugen (vgl. Latour 2006: 269 f.).

In Bezug auf die oben formulierte Annahme, dass methodische Transferprozesse beim Zusammentreffen von Wissenschaftlern unterschiedlicher Forschungsbereiche wie der vor- und frühgeschichtlichen Archäologie, der Kunst- oder der Landesgeschichte zu erwarten wären, kann die Burg mit Peter Galison (1996) als „trading zone“ begriffen werden. Verschiedene Wissenschaftssprachen und Arten des Sehens trafen bei der Untersuchung einer Burg

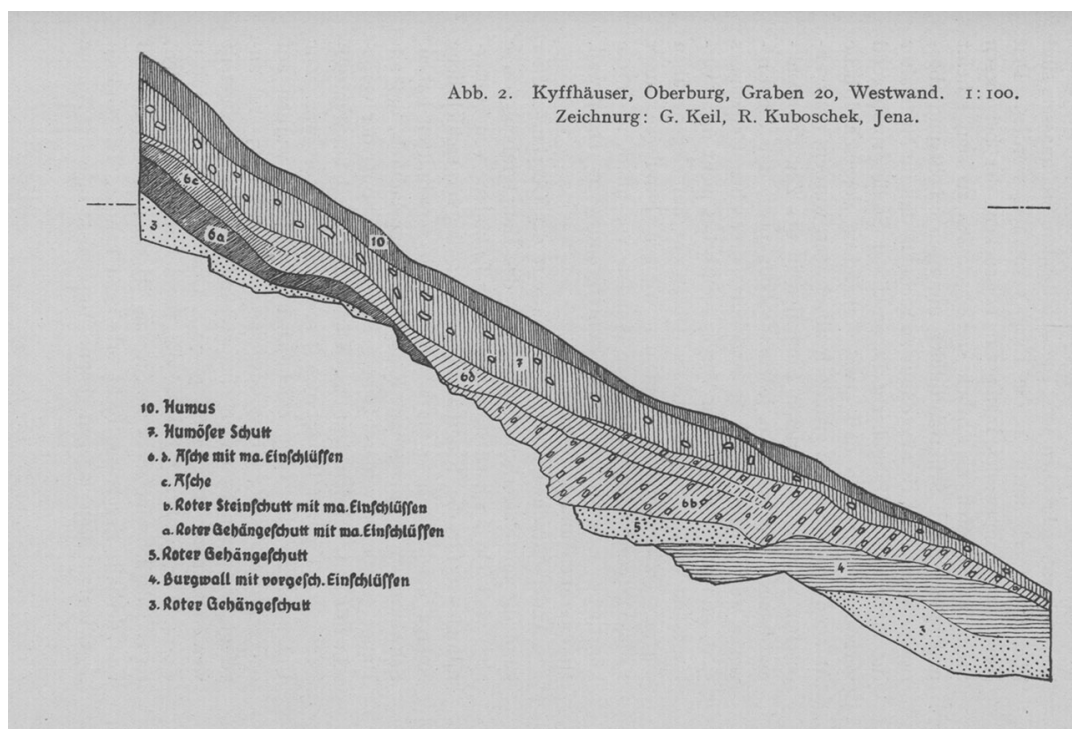

Abb. 4 Zeichnung eines Profils der Ausgrabung der oberen Burgruine auf dem Kyffhäuser Burgberg. Diese Zeichnung basiert auf einer Skizze, die vor Ort angefertigt und dann für den Druck umgezeichnet wurde. Deutlich zu sehen sind die Kulturschichten, die durch unterschiedliche Schraffur zeichnerisch voneinander abgesetzt sind, und die Positionsnummern, die mit den Beschreibungen der jeweiligen Schicht korrelieren (Neumann 1929: Tafel I) 
aufeinander und ließen sich unter gewissen Umständen zusammenführen. Um diese theoretische Annahme empirisch zu stützen, werden nun die konkreten Praktiken der Wissenschaftler bei zwei Burgenforschungsprojekten näher betrachtet.

\section{Forschungspraktiken in den 1930er Jahren}

Im folgenden Abschnitt werde ich anhand zweier Forschungsunternehmen in den 1930er Jahren die Praktiken der archäologischen sowie kunst- und landeshistorischen Feldforschung analysieren. Beide Unternehmen wurden von Wissenschaftlern bestritten, die mit den VFG in Verbindung standen. Beim ersten Unternehmen handelt es sich um die Ausgrabung und bauhistorische Untersuchung der Burg Trifels in der Pfalz, die von Burgenforscher Bodo Ebhardt und dem Archäologen und Historiker Friedrich Sprater durchgeführt wurde. Im zweiten Fall handelt es sich um die Ausgrabung der Burgruine Alt-Tierstein im Kanton Aargau in der deutschsprachigen Schweiz unter der Leitung des Historikers, Archivars und „Westforschers“ Hektor Ammann.

\section{Die Burg Trifels in der Pfalz}

Im Rahmen eines vom bayerischen Ministerpräsidenten Ludwig Siebert ins Leben gerufenen Kulturprogramms für die Rettung mittelalterlicher Burgen, Schlösser und Ruinen in Bayern und in der Pfalz sollte die oberhalb des pfälzischen Städtchens Annweiler gelegene Burg Trifels nicht bloß restauriert, sondern im „staufischen Geist" neu gebaut werden. ${ }^{10}$ Dieses Unternehmen nahm einen herausgehobenen Platz in Sieberts Kulturprogramm ein, der den Wiederaufbau einer der bekanntesten Burgen Deutschlands als eine besondere Kulturleistung des NS-Regimes herausstellen und propagandistisch vermarkten wollte. Der Trifels war für Siebert „der allergrößte Zeuge deutscher Geschichte“ (Stein 1975: 388; Backes 2000: 118). Dem Plan nach sollte vor Beginn der Bauarbeiten der gesamte Burgberg mit dem noch erhaltenen mittelalterlichen Mauerwerk wissenschaftlich untersucht werden. Im März 1937, als Siebert eine Sitzung mit den Denkmalpflegern und Wissenschaftlern, die an diesem Unternehmen beteiligt waren, in München einberief, wurde festgelegt, dass ,in der ganzen Umgebung des obersten Bergkegels allen Spuren der ehemaligen Anlage, Befestigung, Wegzugänge durch Grabungen nachgegangen [werden soll]. “11

Als Leiter seines 1933/1934 begonnenen Burgen- und Schlössersanierungsprogramms hatte Siebert den Architekten, Oberregierungsbaurat Bayerns und Heimatschützer Rudolf Esterer beauftragt (Fleischner 1999: 4). Friedrich Sprater, Archäologe, Historiker und Direktor des „Historischen Museums der Pfalz" in Speyer, sollte die wissenschaftlichen Arbeiten leiten. 
Zusätzlich wurde Bodo Ebhardt, der damals als Deutschlands bekanntester Burgenexperte galt, hinzugezogen. Ebhardt übernahm die bauhistorische Untersuchung der Burg Trifels. Siebert behielt sich die „endgültige Entscheidung in allen Punkten" vor, auch in wissenschaftlichen Belangen. ${ }^{12}$ Aus den erhaltenen Quellen geht hervor, dass Siebert weit höheren Wert auf den Neubau des Trifels legte als auf die wissenschaftlichen Untersuchungen, worin er von den Denkmalpflegern unterstützt wurde. ${ }^{13}$ Erstes Ziel im Unternehmen war, ,aus geschichtlichen, vaterländischen und heimatlichwirtschaftlichen Gründen [...] eine monumentale Gedenkstätte des deutschen Kaisertums und deutschen Verteidigungswillens in der Westmark" zu errichten. ${ }^{14}$ Für die wissenschaftlichen Arbeiten stellte Siebert einen Betrag von 18.000 RM zur Verfügung. Verglichen mit den Kosten des Neubaus ist dieser Betrag als relativ gering einzustufen. ${ }^{15}$

Zunächst befreite die Bau- und Ausgrabungsmannschaft das gesamte Gelände von Gestrüpp und Bäumen. Es wurden Gerüste für die Dokumentation des Mauerwerks gestellt, Vermessungsgeräte installiert und eine Hütte zum Lagern der Instrumente sowie ein Büro für die Bauleitung und die Wissenschaftler errichtet. Die Baumannschaft stellte sodann einen Kran für das Heben der schweren Bau- und Gerüstmaterialien im Burgareal auf. Als nächstes vermaßen die Techniker das gesamte Gelände und hielten die Messergebnisse auf ihren Plänen fest. Das nun auf Millimeterpapier aufgezeichnete Burgareal konnte dadurch in einzelne Ausgrabungssektoren unterteilt werden. Nebst einem Gerüst mit angehängter Rampe zum Abführen von Schutt und Steinen, ${ }^{16}$ verlegten die Arbeiter im unteren Bereich der Burg ein Schienensystem, auf dem mit Loren die Baumaterialien nach oben befördert werden konnten. Diese ganzen Installationen waren sehr aufwändig, das ganze Unternehmen gestaltete sich entsprechend kostspielig.

Ebhardts und Spraters Ziel war, alle durch die Ausgrabungsmannschaft zutage geförderten archäologischen Artefakte zu bergen und zu dokumentieren, um sie wissenschaftlich auszuwerten und als Museumsstücke aufzubewahren. Besonderes Augenmerk musste auf solche Funde gelegt werden, die Datierungshinweise aufwiesen. Dabei sollten vor allem der ursprüngliche Zugangsweg in die Burg und die Zugänge innerhalb des Burgareals sowie die bislang unbekannte tatsächliche Ausdehnung der Burg freigelegt werden. Aufgrund von früheren Sondierungen Spraters und der bisherigen Forschungsliteratur zur Burg erwarteten die Wissenschaftler mindestens zwei große mittelalterliche Bau- und Ausbauphasen, die erste unter den Saliern (etwa 10. bis 12. Jahrhundert), die andere unter den Staufern im 13. Jahrhundert. ${ }^{17}$ Die Fundstücke sollten „nummeriert werden mit Bezeichnung der Fundstelle, Datum usw., wichtige Stücke sind photographisch und zeichnerisch aufzunehmen [...]. Die Fundstellen sind in die Pläne einzutragen." ${ }^{18}$ Die Artefakte sollten so dokumentiert werden, dass sie den verschiedenen Ausgrabungssektoren später zugeordnet werden konnten. 
Zusätzlich forderten Ebhardt und Sprater das Führen eines Fundverzeichnisses und eines Grabungstagebuchs. ${ }^{19}$ Diese methodischen Ansprüche sind gemessen an den oben dargestellten Standards in den frühen 1930er Jahren als relativ weit entwickelt zu beurteilen.

Die von Sprater und Ebhardt angestrebten Ziele konnten zwar recht bald erreicht werden: mehrere Gebäude waren innerhalb und außerhalb des Burgareals zum Vorschein gekommen und die Zugangswege konnten ebenfalls freigelegt werden. ${ }^{20}$ Aber die methodischen Ansprüche, die Sprater und Ebhardt gestellt hatten, wurden bei weitem nicht erfüllt oder konnten nicht in die Praxis umgesetzt werden. Die heute noch erhaltene Dokumentation der wissenschaftlichen Arbeiten lässt nicht darauf schließen, dass jemals ein Fundverzeichnis oder ein Grabungstagebuch existiert hatten. Erhalten sind lediglich Grundrisspläne und Seitenansichten der vermessenen Burg, steingerechte Aufnahmen des Mauerwerks und der architektonischen Details, eine Reihe von Fotografien von Befunden und Fundobjekten (Meyer 2001: 162 f.) sowie Berichte zum Stand der wissenschaftlichen Arbeiten, die Sprater wöchentlich oder monatlich verfasst hatte. Die Ausgräber sind nicht nach der stratigrafischen Methode vorgegangen, sondern haben sich daran orientiert, möglichst rasch neue Befunde zutage zu fördern. Dadurch konnten die Fundobjekte nur grob den einzelnen Grabungssektoren zugewiesen werden, was im Hinblick auf den Erkenntnisgewinn nahezu wertlos war. Eine präzise Rekonstruktion der verschiedenen Bau- und Besiedlungsphasen des Burgbergs war mit diesen Unterlagen kaum möglich. Neuere naturwissenschaftliche Methoden und Techniken, wie die eingangs erwähnten archäobiologischen Methoden oder die Mörtelanalyse, sind nicht angewendet worden. Es findet sich auch kein einziger publizierter oder unveröffentlicht gebliebener Versuch, die archäologischen Funde nach typologischen Gesichtspunkten auszuwerten. Die erhaltene fotografische Dokumentation zeigt, dass Sprater zwar den Überblick über die Befunde behielt und in seine bereits im Voraus erwarteten Bauphasen einordnete, jedoch keine Analyse der Fundobjekte leistete. ${ }^{21}$

Diese Sachlage resultierte zum einen aus der vollständigen Abhängigkeit der Wissenschaftler von Siebert, der, unterstützt darin von den Denkmalpflegern, die Wissenschaftler unter Druck setzte. Die wissenschaftlichen Arbeiten liefen nicht in der Weise ab, dass zunächst in aller Ruhe die Burg untersucht werden konnte und erst danach mit den Bauarbeiten begonnen wurde. Vielmehr forcierten Siebert und Esterer den Neubau, was zur Folge hatte, dass die Wissenschaftler bei ihren Arbeiten die Baumannschaft im Rücken hatten, die auf ein schnelles Vorgehen drängte. Zudem war der Betrag von 18.000 RM für die wissenschaftlichen Arbeiten bereits Anfang Oktober 1937 aufgebraucht, ${ }^{22}$ ohne dass die Untersuchungen abgeschlossen waren. Nach der Bitte Spraters um zusätzliche finanzielle Mittel für die wissenschaftlichen Arbeiten stellte Siebert weitere 5.000 RM zur Verfügung, ${ }^{23}$ ließ Sprater aber wissen, dass er die Fortsetzung der Untersuchungen nur 
widerwillig finanzierte. Die vom NS-Politiker produzierte und den Denkmalpflegern und Wissenschaftlern vermittelte Knappheit an finanziellen Mitteln hatte zur Folge, dass die Denkmalpfleger die Wissenschaftler sabotierten und bei Siebert anschwärzten. ${ }^{24}$ Ebhardt beschwerte sich bei mehreren Gelegenheiten über die mangelnde Zusammenarbeit mit Esterer. ${ }^{25}$

Dieses ungleiche materielle Verhältnis hatte entscheidende Auswirkungen auf die Produktion archäologischen und bauhistorischen Wissens. Sprater ließ mehrheitlich nicht flächig nach Schichten graben, sondern Suchschnitte an jenen Stellen anlegen, an denen er entsprechende Aufschlüsse vermutete, ${ }^{26}$ denn er sah sich in die Situation versetzt, rasche Erfolge zu erzielen, um diese an Siebert weiterzuleiten. So berichtete er, die Ausgrabungen hätten „vollständig neue Erkenntnisse über den Umfang der Burg und über ihre Baugeschichte erbracht." Es seien nicht nur zwei große Bauphasen festzustellen, sondern drei, und zwar eine salische, eine hohenstaufische und eine frühneuzeitliche, die aufgrund eingravierter Jahreszahlen ins 16. Jahrhundert datierte. Außerdem vermutete Sprater eine noch ältere Burganlage, die mehrheitlich aus Holz erbaut worden sei, die er chronologisch aber noch nicht genau bestimmen könne. In den Fels gehauene viereckige Löcher gaben Anlass zu dieser Vermutung. ${ }^{27}$ Angesichts dessen, dass hölzerne Burgen im frühen Mittelalter in der Regel auf künstlich aufgeschütteten Erdhügeln errichtet wurden und nicht auf einem Felssporn (vgl. Hinz 1981), was bereits in den 1930er Jahren bekannt war, liegt es nahe, diese Vermutung Spraters mehr strategischen als erkenntnisgeleiteten Überlegungen zuzuschreiben. Sprater wollte dem bayerischen Ministerpräsidenten eine weit zurückreichende Besiedlungsgeschichte des Burgbergs vorspiegeln, in der Annahme, Siebert würde aufgrund seines ideologisch-nationalistischen Hintergrunds damit eine erste Inbesitznahme des Burgfelsens in "germanischer Zeit" assoziieren, was eine Mobilisierung weiterer Forschungsgelder erleichtert hätte.

Die mangelhafte wissenschaftliche Dokumentation ist zum anderen darauf zurückzuführen, dass keine einheitliche Methode angewandt wurde. Ebhardt und Sprater arbeiteten strikt getrennt voneinander, jeder richtete sich nach seinem eigenen methodischen Vorgehen. Dies führte dazu, dass beide Dokumentationen in unterschiedlicher Art ausgeführt wurden und nicht miteinander harmonisiert werden konnten. Im Gegensatz zu Sprater arbeitete Ebhardt nicht mit einem Positionsnummernsystem und beschrieb die gezeichneten Befunde nicht. Die steingerechten Aufnahmen des Mauerbestands schienen für ihn in Bezug auf die Interpretation der Befunde selbstredend gewesen zu sein. Für Ebhardt, der als gelernter Architekt auch Burgen und Schlösser wiederaufbaute (vgl. Fischer 2010), standen ästhetische und künstlerische Aspekte im Vordergrund und nicht so sehr das Erstellen einer methodisch ausgefeilten Dokumentation. Sprater dagegen führte die getroffenen Beobachtungen in seinen Berichten zwar zusammen, eine 
detaillierte Dokumentation der Grabungsbefunde und Fundobjekte hat aber auch er nicht erstellt.

Der Ausstoß an publizierten Auswertungen des empirischen Materials war entsprechend gering und bis auf einige wenige populäre Veröffentlichungen (Sprater 1937 und 1945) wurden die Forschungen auf der Burg Trifels nicht im Rahmen einer größeren Publikation der Öffentlichkeit zugänglich gemacht. ${ }^{28}$ Allerdings war ein Buch geplant, in dem alle am Unternehmen beteiligten Akteure und weitere Forscher, die nicht direkt in die archäologischen und bauhistorischen Untersuchungen eingebunden waren, sich jedoch mit der Burg Trifels beschäftigten, Beiträge einbringen sollten. Im Frühjahr 1939 beabsichtigte das zu den VFG gehörende „Saarpfälzische Institut für Landes- und Volksforschung“ (vgl. Freund 2006), eine TrifelsPublikation herauszugeben. Das Buch sollte „für die besondere Lage des Westraumes in der Gegenwart auch im Reich werbend [...] wirken." ${ }^{29}$ Ein Gemeinschaftswerk war also geplant, an dem NS-Kulturpolitiker, Denkmalpfleger, akademische Wissenschaftler und Heimatforscher beteiligt sein sollten. Je ein Kapitel wurde je einem Bearbeiter zugeteilt. Der erste Teil des „Trifels-Werks“ sollte eine geopolitisch orientierte Darstellung des mittelalterlichen Reiches in Westeuropa leisten. ${ }^{30}$ Der zweite Teil sollte nebst den Ausgrabungsergebnissen auch Genealogie, Heraldik und die denkmalpflegerische Neukonzeption der Burg als „Ehrenstätte“ thematisieren. ${ }^{31}$

Die Mitarbeiter des Saarpfälzischen Instituts verstanden ihre Institution als Anlaufstelle für regionale Laiengelehrte und pfälzische Heimatforscher. ${ }^{32}$ Hier wurden die "heimatlichen“ Forschungen „aus dem Volk“ vereint. Im Vordergrund stand die Absicht, aus akademischen Wissenschaftlern und Heimatforschern eine Arbeitsgemeinschaft zu bilden. ${ }^{33}$ Hierbei handelte es sich um ein holistisches Konzept, das die Wissenschaft vom akademischen Elfenbeinturm wieder zum „Volk“ zurückführen sollte. Dieses Leitmuster wird anhand der Struktur des Saarpfälzischen Instituts deutlich, in dem Wissenschaft, Öffentlichkeit und Kulturpolitik zusammenkamen. Ungleich den Landesdenkmalämtern unterstand es nicht einem Kultusministerium, sondern der neu geschaffenen Gaukulturleitung. ${ }^{34}$ Gaukulturwart Kurt Kölsch hatte bereits 1933 das Ziel einer „Zusammenfassung aller wissenschaftlichen Vereine der Pfalz und die Umwandlung des Vereins Historisches Museum der Pfalz e. V. [...] in die Arbeitsgemeinschaft der völkischen Wissenschaft im Kampfbund für deutsche Kultur in der Westmark" verfolgt. Diese pfälzische Arbeitsgemeinschaft der „völkischen Wissenschaft" war damit in den „Neubau des nationalsozialistischen Staates einbezogen und [...] auf die [...] Aufgabe verpflichtet, die Zusammenhänge zwischen Blut und Boden, Rasse

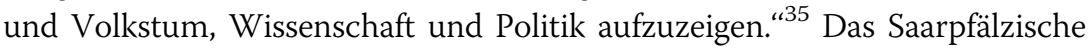
Institut beabsichtigte nicht nur, eine "ganzheitliche Wissenschaftspraxis“ zu etablieren, sondern vor allem eine politische im Sinne des Nationalsozialismus, was einer Verschmelzung von Politik und Wissenschaft gleichkam. Dies 
bedeutete nun nicht, dass das "Trifels-Werk“ zumindest potentiell keine innovativen Ergebnisse erbracht hätte. Abgesehen davon, dass sich die Herausgeber mit den Autoren über den Stil der Gemeinschaftsmonografie - rein populärwissenschaftlich oder akademisch? - nicht einigen konnten, ${ }^{36}$ geriet das Unternehmen bereits im Spätjahr 1939 ins Stocken. Aufgrund der politischen Spannungen in Westeuropa wurden die Herausgeber angewiesen, ihren Plan vorerst zurückzustellen. Nach dem erfolgten Westfeldzug durch das NS-Regime wurden dann zahlreiche am Unternehmen beteiligte Mitarbeiter in die Wehrmacht eingezogen, was dazu führte, dass das Buchprojekt während des Zweiten Weltkriegs auf Eis gelegt und später nie wieder aufgenommen wurde. ${ }^{37}$

\section{Die Ausgrabung der Burgruine Alt-Tierstein}

In der deutschsprachigen Schweiz fanden in den 1920er und 1930er Jahren zahlreiche Untersuchungen von mittelalterlichen Monumenten statt, wobei Burgruinen eine besondere Aufmerksamkeit zukam. Seit dem späten 19. Jahrhundert erforschten und konservierten Heimat- und Burgenvereine, Privatgelehrte und akademische Forscher, Archivare sowie kantonal und landesweit agierende Organisationen für Denkmalpflege vermehrt Schlösser, Burgen und Ruinen. ${ }^{38}$ Ähnlich wie in Deutschland waren diese Objekte Träger kultureller Erinnerung. Intensiver noch als in Deutschland wurde in der deutschsprachigen Schweiz für wissenschaftliche Untersuchungen und Restaurierungsarbeiten dieser Bauwerke der sogenannte Freiwillige respektive der Studentische Arbeitsdienst in Anspruch genommen. In manchen Kantonen existierte seit 1934 gar ein Archäologischer Arbeitsdienst, der exklusiv für archäologische und denkmalpflegerische Arbeiten eingesetzt wurde. ${ }^{39}$ Der Arbeitsdienst verschaltete die Erforschung und Bewahrung vaterländischer Altertümer mit seinem Erziehungsauftrag, der dahingehend lautete, die meist männlichen arbeitslosen Jugendlichen vor Alkoholmissbrauch und Kriminalität zu schützen, indem ihnen eine kulturell sinnvolle Arbeit angetragen wurde, die gleichzeitig erzieherisch im nationalen Sinne wirkte. ${ }^{40}$

Einige deutschschweizerische Denkmalpfleger und Wissenschaftler standen mit den VFG in Verbindung, insbesondere mit der so genannten Westdeutschen und der Alpenländischen Forschungsgemeinschaft. ${ }^{41}$ Eine zentrale Rolle spielte dabei der Archivar des Staatsarchivs des Kantons Aargau Hektor Ammann, der seit den 1920er Jahren als Bindeglied zwischen schweizerischen Wissenschaftlern und der völkischen kulturwissenschaftlichen Gemeinschaftsforschung in Deutschland agierte (Fahlbusch 2008; Simon 1995). Von 1934 bis 1935 leitete Ammann zusammen mit Archäologen, Denkmalpflegern und burgenbegeisterten Lokalpolitikern die Ausgrabung und Konservierung der Burgruine Alt-Tierstein im Kanton Aargau (Ammann 1935: 4). Finanziert wurde das Unternehmen durch Beiträge lokaler und regionaler Heimatschutz-, Geschichts- und Archäologievereine sowie privater 
Gönner. Etwa vierzig Prozent der Kosten wurden vom Bund und vom Kanton getragen. Sechs Monate lang und dann im Frühjahr 1935 noch einmal während zehneinhalb Wochen wurden mit einer Mannschaft aus durchschnittlich 48 jungen Männern im Rahmen eines „Arbeitslagers“ die Ausgrabungs- und Konservierungsarbeiten durchgeführt. Wissenschaftlicher Leiter war Hans Erb, ein junger Archäologe, der kurz vor dem Abschluss seines Studiums stand (Ammann 1935: 5 f.). ${ }^{42}$

Ähnlich wie bei der Burg Trifels arbeiteten auch im Falle der Burgruine Alt-Tierstein Vertreter außerakademischer Kulturvereine, lokale und regionale Kulturpolitiker, Historiker und Bibliothekare sowie Archäologen gemeinsam an der Konzeption und Durchführung dieses Unternehmens (Ammann 1935: 6). Erb, ein geschulter und trotz seiner jungen Jahre in der Schweiz kein unbekannter Archäologe (Dubler et al. 2006), sandte jede Woche einen Bericht an den Grabungsausschuss. Er sah sich dabei vor eine schwierige Aufgabe gestellt, denn im Laufe der Arbeiten hatte sich gezeigt, dass unter den mittelalterlichen Schichten vorgeschichtliche Siedlungshorizonte lagen (Erb 1935: 29). Zudem zeichneten sich mehrere Bauphasen der mittelalterlichen Burg ab, deren historische Entstehung in einen logischen Zusammenhang gebracht werden musste. Um diesen komplexen Befund zu dokumentieren, unterteilte Erb die Ausgrabungsfläche in mehrere Parzellen. In jeder dieser Teilflächen ließ er zuerst einen ein Meter breiten, „bis auf den gewachsenen Boden [d. i. der anstehende Boden] geführten Sondiergraben" ausheben, um einen Einblick in die Schichtenverhältnisse" zu gewinnen, sodass er bei der Ausgrabung der Gesamtfläche dem Verlauf der Schichten verlässlich folgen konnte (Erb 1935: 18). Er führte ein Grabungstagebuch und erstellte Tagesberichte, ließ die Befunde von technischen Hilfskräften fotografieren und Zeichnungen der Mauerzüge und von einzelnen Profilen anfertigen (Erb 1935: 16). Aus den wöchentlichen Berichten geht deutlich hervor, dass Erb die Funde den Kulturschichten zuordnete und im Befundzusammenhang detailliert beschrieb. Die Kleinfunde ordnete er typologisch nach Stil, Funktion und Material, wie das in der vor- und frühgeschichtlichen Archäologie üblich war (Erb 1935: 64-71). ${ }^{43}$ Auch in seinem abschließenden Bericht über die Ausgrabungsergebnisse ging Erb ausgesprochen systematisch vor. Zuerst beschrieb er sein methodisches Vorgehen. Dann ging er zur Beschreibung und Analyse des Grabungsbefunds und der archäologischen Funde über und versuchte, beide Komplexe miteinander zu verknüpfen (vgl. Erb 1935).

Die hier angewandte Methode war im Vergleich zum Trifels-Unternehmen weit ausgefeilter, wofür zwei Umstände ausschlaggebend waren. Zum einen standen Erb sowohl genügend Arbeiter als auch Zeit zur Verfügung, zwei entscheidende Faktoren, die bei den wissenschaftlichen Arbeiten an der Burg Trifels nicht gegeben waren. Zum anderen wurde zur gleichen Zeit und in der Nähe der Burgruine Alt-Tierstein ein prähistorischer und römischer Siedlungsplatz ausgegraben. Der dortige Ausgrabungsleiter war Gerhard 
Bersu, ein deutscher Archäologe in leitender Position bei der „RömischGermanischen Kommission“, der als einer der angesehensten Ausgräber seiner Zeit galt (vgl. Krämer 2002). Bersu fungierte als Inspirator für die Schweizer Archäologen, die bei mehreren Gelegenheiten die in der Schweiz bis in die 1930er Jahre vorherrschenden, im Vergleich zu Deutschland rückständigen Methoden bemängelt hatten. ${ }^{44}$ Erb (1935: 19 f.) konstatierte, dass die archäologische Untersuchung der Burgruine Alt-Tierstein „die erste Grabung in unserem Lande [ist], bei der die exakten prähistorischen Grabungsmethoden, die einen Hauptakzent auf die Schichtenbeobachtung legen, an einem mittelalterlichen Objekt eingehend erprobt wurden." Es seien „Beobachtungen gemacht und Schlüsse für eine exakte Verarbeitung des Tatsachenmaterials ermöglicht worden, die bei einer Grabung, die lediglich die Freilegung der verschütteten Mauerzüge im Auge gehabt hätte, übersehen worden wären." Erb sprach diesen „exakten Methoden“ der vor- und frühgeschichtlichen Archäologie das eigentlich innovatorische Potential für die Erforschung mittelalterlicher Burgruinen zu. So war es ihm möglich, ganze sieben Bauphasen der Burgruine festzumachen, die er aufgrund der Verknüpfung von datierender mittelalterlicher Keramik mit den Baubefunden vom 12. bis zum 15. Jahrhundert zuerst in ihrer Abfolge aufschlüsselte und anschließend die Datierung der Schichten und Fundobjekte vornahm (Erb 1935: 89-94).

Bezüglich der Frage nach Anwendung eines interdisziplinären Methodenkanons bei der Untersuchung der Burgruine Alt-Tierstein sind zwei Punkte festzuhalten. Erstens waren nicht mehrere Wissenschaftler aus unterschiedlichen Disziplinen an der Untersuchung direkt beteiligt. Sicher standen Historiker wie Ammann unterstützend zur Seite. Erb führte aber die Ausgrabungsarbeiten in Zusammenarbeit mit Archäologen wie Bersu durch, ohne dass er mit Kunsthistorikern (Befundaufnahme an den Mauern) oder Volkskundlern (Alltag auf mittelalterlichen Burgen) kooperierte. Er selbst betrieb keine volkskundlichen Studien und führte auch keine kunsthistorischen Analysen der erhaltenen Bauplastik durch, vielmehr ordnete er die Bautechnik der Mauern nur grob den hoch- bis spätmittelalterlichen Epochen zu (Erb 1935: 47, 73-75, 93). Ihm war an der detaillierten und „objektiv“ archäologisches Wissen versprechenden Dokumentation gelegen. Erb konzentrierte sich daher auf die Untersuchungsgegenstände, mit denen er als Archäologe vertraut war. Während beim Trifels-Unternehmen mehrere Wissenschaftler zwar in Absprache mit-, methodisch jedoch getrennt voneinander arbeiteten, gab bei der Untersuchung der Burgruine Alt-Tierstein ein einziger Wissenschaftler die methodischen Richtlinien vor, nach denen die Befunde erfasst wurden.

Zweitens ist anzumerken, dass bei näherer Betrachtung des „Arbeitslagers Tierstein" nicht eindeutig zu bestimmen ist, ob wissenschaftliche Faktoren tatsächlich entscheidend waren für das Leitungsgremium und die Geldgeber 
des Unternehmens. Bei den Arbeiten an der Burgruine Alt-Tierstein waren die Wissenschaftler eng an die Vorgaben der Leitung des Arbeitsdienstes gebunden, die nebst wissenschaftlichen Zielsetzungen auch einen Erziehungsauftrag verfolgte. $\mathrm{Zu}$ diesen volkserzieherischen Aspekten gehörte, dass physische Arbeit, wie sie bei der Ausgrabung der Ruine Alt-Tierstein verrichtet wurde, im Geist der sozialtechnisch-völkischen Utopie eines „gesunden Volkskörpers“ die jungen Männer körperlich stärken sollte. Dies wird auf einem Werbeplakat des Archäologischen Arbeitsdienstes der Schweiz deutlich, auf dem ein junger Mann mit entblößtem, muskulösem Oberkörper abgebildet ist, in der einen Hand einen Pickel, in der anderen den Hals einer Amphore (Abb. 5). ${ }^{45}$

Ein Indiz dafür, dass bei diesem Unternehmen mindestens zu gleichen Teilen volkserzieherische Absichten im Vordergrund standen, war, dass zu wenige wissenschaftliche Kräfte vor Ort waren, um die Arbeiten adäquat zu betreuen. Tatsächlich war Hans Erb der einzige Wissenschaftler auf der Ausgrabung. Er wurde zwar regelmäßig von den Mitgliedern des Grabungsausschusses besucht und erhielt Unterstützung, letztlich musste er sich aber allein um die durchschnittlich 48 Arbeitsdienstmänner kümmern. Dies allerdings ist für einen einzelnen Wissenschaftler nahezu unmöglich. So berichtete Erb (1935: 18) in der abschließenden Publikation, dass er für die „Grabarbeiten [...] einen Mittelweg zwischen sehr sorgfältiger Arbeit mit eingehender und ständiger Beobachtung einerseits und dem bei den großen zu bewältigenden Schuttmassen naheliegenden Streben nach Kubikmeterarbeit andererseits" changieren musste. Zwischen den Zeilen steht, dass bestimmte Befunde durch das unbeaufsichtigte Vorgehen der in der feinen archäologischen Arbeit ungeübten Arbeitsdienstmänner verlorengegangen waren. Die starke Fokussierung auf technisch avancierte Ausgrabungsmethoden kann daher auch als Minimallösung gesehen werden, um einerseits gute Ergebnisse zu erzielen, andererseits die Anstellung von Wissenschaftlern auf ein Minimum zu beschränken. Das schon bei der Ausgrabung der Burg Trifels beobachtete Auseinanderklaffen von Anspruch und tatsächlicher Umsetzung war demnach auch charakteristisch für die Untersuchungen der Burgruine Alt-Tierstein.

Damit im Zusammenhang stand die bei diesem Burgenforschungsprojekt ausgesprochen wichtige Öffentlichkeitsarbeit. Denn das „Arbeitslager Tierstein “ war das größte Unternehmen dieser Art in der Schweiz. ${ }^{46}$ Wie Ammann $(1935: 7,9)$ festhielt, war dem Grabungsausschuss ausgesprochen wichtig, der Öffentlichkeit zu demonstrieren, dass hier „eine der bedeutendsten Burgruinen des Kantons" ausgegraben wurde und dass bei den Konservierungsarbeiten lokale Handwerker beschäftigt werden konnten. Eines der zentralen Anliegen bei dem Unternehmen war, dass das Fricktal im Kanton Aargau „auf dem Tierstein ein sehenswertes Denkmal seiner Vergangenheit [...] und zugleich auch einen sehr schönen Aussichtspunkt" 
erhalten sollte (Ammann 1935: 9). Im Zentrum der Ausgrabung und Konservierung der Ruine Alt-Tierstein stand demnach primär die Durchführung eines gemeinschaftlich-heimatlichen Unternehmens. Dies resultierte zu einem Gutteil aus einer Popularisierungsstrategie der Akteure. Ammann und seine Mitstreiter waren daran interessiert, kulturwissenschaftlichen Forschungsunternehmen breite öffentliche Aufmerksamkeit zu verschaffen, um weitere Gelder vom politischen und öffentlichen Feld mobilisieren zu können. Hierbei muss auch bedacht werden, dass die vor- und frühgeschichtliche Archäologie zu diesem Zeitpunkt in der Schweiz noch keine akademisch etablierte Disziplin war. Das erste Institut für Vor- und Frühgeschichte wurde erst 1943 an der Universität Basel eingerichtet (Laur-Belart 1943). Die Akteure verfolgten mit dem Kulturunternehmen auf der Burgruine Alt-Tierstein also nicht nur ein wissenschaftliches Ziel, sondern auch ein kultur- und wissenschaftspolitisches. Diese beiden Zielsetzungen schlossen sich grundsätzlich nicht aus, führten im vorliegenden Fall aber zu einer Zurückstellung der wissenschaftlichen Anliegen.

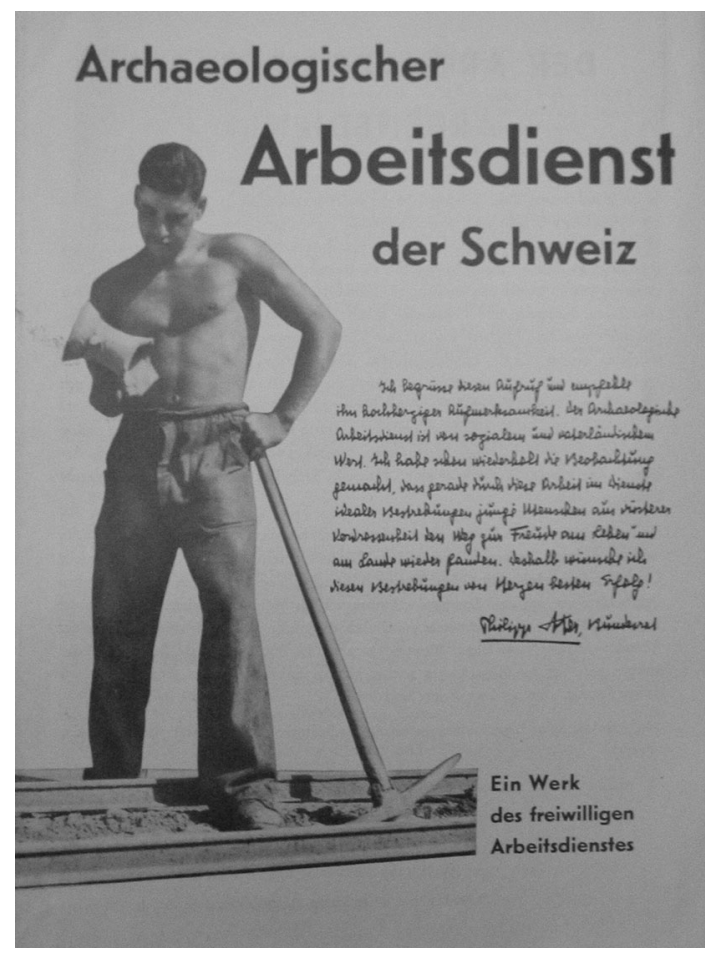

Abb. 5 Titelblatt einer Werbebroschüre für den „Archäologischen Arbeitsdienst der Schweiz" 


\section{Disziplinäre Gebundenheit von Methoden und Forschungsobjekten}

Die beiden Beispiele kulturwissenschaftlicher Feldarbeit verdeutlichen, dass für die 1930er Jahre keine Verdichtung der Praktiken zu einer einheitlichen Vorgehensweise zu beobachten ist. Wie die Untersuchungen der Burg Trifels zeigten, arbeiteten die Wissenschaftler methodisch voneinander getrennt, bei den archäologischen Arbeiten an der Burgruine Alt-Tierstein unterstellte der Grabungsleiter Hans Erb die zu untersuchenden Objekte den "exakten“ Methoden der vor- und frühgeschichtlichen Archäologie, ohne dabei andere Methoden zu berücksichtigen. Bei beiden Unternehmen standen zudem nicht nur wissenschaftliche Aspekte im Vordergrund, sondern auch kulturpolitische und volkserzieherische. Dieser Sachverhalt wird durch zwei wissenschaftssoziologische Befunde untermauert, die ich im Folgenden erläutern möchte.

Erstens waren in der archäologischen Mittelalterforschung in den 1930er Jahren die epistemischen Objekte nur unzureichend semantisch festgelegt. Dies hatte entscheidenden Einfluss auf die damit im Zusammenhang stehenden Praxissysteme. Archäologen, Kunst- und Landeshistoriker verstanden nicht dasselbe unter dem Begriff ,Burg. Noch 1941 stellte der Burgengeograf Carl Storm (1941: 119) fest, es sei in der Vergangenheit zwar „verschiedentlich versucht worden, diesen Begriff festzulegen. Aber bis zur Stunde versteht der Burgenkundler etwas anderes unter der Burg als der Namensforscher, und der Siedlungshistoriker und Vorgeschichtler pflegt ihn wesentlich weiter zu fassen, als der Geograph ihn gerne festgelegt haben möchte." Laut Storm liege dies daran, „dass das Gebiet der Burgenkunde und Geographie eben von den verschiedenen Wissenschaftszweigen her angefasst wurde, die aber erst jetzt $\mathrm{zu}$ ahnen beginnen, wo hinaus und zu welchen Untersuchungskreisen und Zielen der Weg führt.“

Die Plausibilität dieser Feststellung zeigt sich an den Forschungskooperationen, die sich der Untersuchung von Burgen widmeten. Zunächst ist festzuhalten, dass in den 1930er Jahren keine Arbeitsgemeinschaft für kulturwissenschaftliche Mittelalterforschung als solche existierte. Dagegen existierte seit den frühen 1930er Jahren eine Arbeitsgemeinschaft, deren Mitglieder ausschließlich Pfalzen, also frühmittelalterliche Königsresidenzen, in Westdeutschland erforschten. ${ }^{47}$ Auch in Thüringen, Sachsen und Brandenburg gab es im Umfeld der deutschen "Ostforschung" eine ähnliche Arbeitsgemeinschaft, welche die Pfalzen Tilleda, Werla und Goslar archäologisch, kunst- und landeshistorisch untersuchte (vgl. Brandi 1935). ${ }^{48}$ Diese Pfalzenforscher fokussierten ausschließlich auf Objekte, die in eine spezifische Periode datierten, nämlich in karolingische und ottonische Zeit. Auch die 1927 gegründete „Arbeitsgemeinschaft zur Erforschung der nord- und ostdeutschen vor- und frühgeschichtlichen Wall- und Wehranlagen", die ebenfalls im Rahmen der „Ostforschung“ agierte (Grunwald 2009: 56, 66; Halle 2008: 117), 
erforschte ausschließlich Burgwälle. Bei Burgwällen handelte es sich nicht um hochmittelalterliche, steinerne Burgen, sondern um abgegangene, ehemals hölzerne Befestigungen entweder aus der Eisenzeit oder aus dem Frühmittelalter. Beide Forschungsrichtungen betrieben ihrem Selbstverständnis nach Burgenforschung, untersuchten jedoch de facto unterschiedliche Objekte.

Interdisziplinäre Ansätze wurden lediglich im Falle dieser beiden Objektgattungen umgesetzt. Der deutsche Frühmittelalterspezialist Herbert Jankuhn konstatierte 1939, dass bei den Ausgrabungen der Kaiserpfalz Werla „die immer mehr zu wünschende enge Zusammenarbeit zwischen Vorgeschichts- und Bauforscher verwirklicht worden" sei. Die dem Unternehmen entsprungene Monografie erweckt tatsächlich den Eindruck, dass hier interdisziplinär gearbeitet wurde. ${ }^{49}$ Auch im Falle der Burwallforschung berichteten die daran beteiligten Wissenschaftler, dass dort „zum ersten Male Vorgeschichtler und Geschichtsforscher in ständiger Zusammenarbeit zusammen wirkten" (Brackmann 1937: 174). Interdisziplinäre Wissenschaftspraxis existierte nur in solchen Forschungskooperationen, in denen epistemisch eindeutig bestimmte Forschungsobjekte im Fokus standen, die deutlich abgegrenzten historischen Epochen zugeordnet werden konnten, im vorliegenden Fall also Pfalzen und Burgwällen. Hochmittelalterliche Burgen und Burgruinen dagegen gehörten nicht zu den eindeutig bestimmten Forschungsobjekten, sondern waren für eine disziplinär-methodische Zuordnung semantisch zu indifferent.

Zweitens war für die nicht erfolgte disziplinäre Verdichtung in der kulturwissenschaftlichen Mittelalterforschung in den 1930er Jahren die Sachlage entscheidend, dass sich die für die spätere Mittelalterarchäologie wichtigen Disziplinen und Forschungsfelder im Hinblick auf Konsolidierung und akademische Etablierung in unterschiedlichen Entwicklungsstadien befanden. Während die Kunstgeschichte eine akademisch festgefügte Disziplin und die Landesgeschichte eine Subdisziplin der Geschichte waren (vgl. Dilly 1979: 80258; Wallraff 2008), befand sich die vor- und frühgeschichtliche Archäologie in der Situation, noch nicht vollständig an den deutschen und deutschschweizerischen Universitäten etabliert zu sein (Grabolle et al. 2003: 868; Grünert 2002; Pape and Wolfgang 2002). In den 1930er Jahren stand vor- und frühgeschichtlichen Archäologen das Ziel vor Augen, die Wissenschaftlichkeit ihres Fachs mit Hilfe von „exakten“ technischen Methoden zum Zweck der akademischen Institutionalisierung ihrer Wissenschaft zu demonstrieren. Sie waren deshalb nicht um eine Kooperation auf methodischer Ebene mit Historikern und Kunsthistorikern bemüht, sondern um eine „Prähistorisierung“ kunsthistorischer und historischer Forschungsobjekte. Bei genauem Hinsehen schwebte dem eingangs zitierten Bolko von Richthofen keine interdisziplinäre Arbeitsweise vor, vielmehr sah er die mittelalterarchäologische Forschungspraxis als Erweiterung der Vor- und Frühgeschichte. Für ihn war die archäologische Erforschung des Mittelalters zwar eine 
disziplinenübergreifende Wissenschaftskonzeption, allerdings sollten sich die Mittelalterarchäologen „der gleichen Arbeitsweise [...] bedienen wie die Urgeschichtsforschung“. Die Forschungsobjekte des Mittelalters sollten laut Richthofen unter der methodischen Hoheit der vor- und frühgeschichtlichen Archäologie stehen. ${ }^{50}$ Die Archäologen inkorporierten Forschungsobjekte in ihre Disziplin, die traditionell der mittelalterlichen Landesgeschichte oder der außerakademischen Laienforschung zugeschrieben waren, um Deutungshoheit über diese Wissensobjekte zu erlangen. Die daraus hervorgehenden Strategien der Wissenschaftler zielten deshalb auf eine verstärkte Grenzziehung mit Hilfe ihrer „exakten“ prähistorischen Methoden ab und nicht auf eine Überwindung dieser Grenzen.

\section{Konklusion: Zur disziplinären Nichtkonsolidierung „interdisziplinärer Praktiken“"}

Die Herausbildung der Mittelalterarchäologie als ein disziplinär gefestigtes Feld mit einem neuen, interdisziplinären Methodenkanon war nicht schon in den 1930er Jahren erfolgt, sondern muss als eine spätere Entwicklung angesehen werden. Im Gegensatz zu Vor- und Frühgeschichte, „Volksgeschichte“ und Volkskunde hat keine Konsolidierung in der kulturwissenschaftlichen Mittelalterforschung stattgefunden. Angesichts der in der Forschungsliteratur dargelegten disziplinären Verdichtungen unterschiedlicher Forschungsbereiche unter dem Dach der Gemeinschaftsforschung und der oben aufgeführten Evidenzen für die Annahme einer solchen Entwicklung - auch für die kulturwissenschaftliche Mittelalterforschung - erstaunt dieser Befund. Hierfür können zwei Erklärungsansätze angeführt werden.

Zum ersten war das Verständnis dessen, was bei einer Burg genau zu untersuchen war, bei den an solchen Unternehmen beteiligten Wissenschaftlern und Denkmalpflegern zu divergent, als dass eine gemeinsame Kommunikations- und Praxisebene geschaffen werden konnte. Dies lag vor allem an der begrifflichen Unbestimmtheit des epistemischen Objekts ,Burg“ Für Kunsthistoriker bestand eine Burg aus Mauerwerk, Mauerresten und Bauplastik, also Resten von Gewölbebögen oder Fensterfassungen aus Stein. Diese Vorstellungen bestimmten die Praktiken der kunsthistorischen Feldforscher, nämlich die bauhistorische und typologische Analyse dieser materiellen Überreste. Für Archäologen war eine Burg vor allem ein ehemals vorhandenes Bauwerk, das nun verschüttet war und das mit „exakten“ Ausgrabungsmethoden untersucht werden musste. So erklärt sich auch, weshalb diese Methoden vornehmlich bei solchen Objekten zur Anwendung kamen, bei denen wenig Mauerwerk, jedoch eine ansehnliche Anhäufung von Schutt und Kulturschichten vorlag, was bei den frühmittelalterlichen Burgwällen und 
Pfalzen in der Regel der Fall war. Ganz andere Vorstellungen hatten Denkmalpfleger davon, was mit einer Burgruine anzufangen war. Wissenschaft stand hier nicht im Vordergrund, vielmehr ging es ihnen um die Konservierung oder, wie in den 1920er und 1930er Jahren weit verbreitet, um die Revitalisierung einer Burg für kulturpolitische Zwecke oder als Beitrag zur Schaffung einer völkisch-nationalen Erinnerungskultur. Noch einmal anders war das Verhältnis von Volkskundlern und Landeshistorikern zu Burgen und Burgruinen. Für die einen eröffneten die von den Archäologen geborgenen materiellen Überreste einen Einblick in die Alltagskultur, die anderen konzentrierten sich darauf, die Burg auf eine Landeskarte aufzuzeichnen und in den Zusammenhang mit den aus den schriftlichen Quellen eruierten Herrschaftsverhältnissen im Mittelalter zu setzen (vgl. etwa Hotz 1937 und 1940).

Zum zweiten ging aus der strukturellen Lage der an der späteren Mittelalterarchäologie beteiligten Wissenschaften in den 1930er Jahren keine Notwendigkeit eines neuen interdisziplinären Wissenschaftsgefüges hervor. Wie eingangs erwähnt, war für die disziplinäre Verschiebung der traditionellen Chemie zur Biochemie ein methodisches Krisenmoment in der Chemie ausschlaggebend (vgl. Reinhardt 2006). Die Chemie befand sich zur Zeit ihrer Umwandlung in einer ganz anderen Situation als die hier behandelten Wissenschaften, denn ihr etablierter Methodenkanon hatte sich gewissermaßen totgelaufen. Die für den Methodenkanon der späteren Mittelalterarchäologie entscheidende vor- und frühgeschichtliche Archäologie war in den 1930er Jahren dagegen eine Disziplin, die erst im Begriff war, sich an den Universitäten $\mathrm{zu}$ institutionalisieren. Entscheidend im Kampf um Positionen an den Universitäten war für Archäologen die Demonstration der Überlegenheit ihrer „exakten“ technischen Methoden. Diese Strategie verfolgten vor- und frühgeschichtliche Archäologen auch gegenüber ihren Nachbardisziplinen, was sich bei den beiden besprochenen Beispielen dahingehend äußerte, dass Archäologen nicht daran interessiert waren, die Methoden und Ansätze von Volkskundlern, Kunst- und Landeshistorikern in ihren Methodenkanon einzubauen. Vielmehr intendierten sie, bestimmte Forschungsgegenstände diesen Disziplinen zu entreißen und zu „archäologisieren“. Dies führte letztlich dazu, dass eine Zusammenarbeit nur in solchen Fällen und bei solchen Untersuchungsobjekten zustande kam, bei denen die Archäologen methodisch gesehen die Oberhand hatten. Von einer gleichberechtigten interdisziplinären Zusammenarbeit kann bei solchen Unternehmen kaum gesprochen werden.

Diese Befunde zeigen, dass die wissenschaftssoziologische und praxeologische Analyse von Veränderungen und Verschiebungen disziplinärer wissenschaftlicher Gefüge zu durchaus anderen Ergebnissen führt als konzeptgeschichtlich angelegte Untersuchungen. Dies wirft die Frage auf, ob eine mikrohistorische Erforschung der Praktiken jener Wissenschaftler, die an dem völkisch-revisionistischen Unternehmen der „Volksforschung“ und 
„Volksgeschichte“ beteiligt waren, zu einer anderen Interpretation führen könnte, als dies mehr konzepthistorisch orientierte Analysen vorgeschlagen haben, wonach diese „interdisziplinären“ Forschungskooperationen die methodischen Grundlagen der westdeutschen Struktur- und Sozialgeschichte der 1950er und 1960er dargestellt haben (vgl. Oberkrome 1991 und 1993).

\section{Anmerkungen}

1 Zur Definition von Interdisziplinarität vgl. Jungert 2010 und Kocka 1987. Zur Kritik von Inter- und Transdisziplinarität vgl. Weingart, Carrier \& Krohn 2007: 196.

2 Landeshauptarchiv Koblenz (LHAKo), Bestand 403, Nr. 15016: Veranstaltungen in den Rheinlanden anlässlich der Jahrtausend-Feiern, zusammengestellt durch den Rheinischen Verkehrsbund e. V., Sitz Bad Godesberg, datiert Juni 1925.

3 Vgl. Schweizerisches Burgenarchiv (Schweiz. Burgenarchiv), Akte „Dokumentationen zu Burg Homburg, Burg Bischofstein, Farnsburg, Rapperswil, Wartenberg“.

4 Bundesarchiv Berlin (BA Berlin), R 43 II/1260, Bl. 25 f.: Georg Lill an das Staatsministerium des Innern z. Hd. des H. Ministerialrats Jacob, 16.9.1935; ebd., Bl. 62: Hans Heinrich Lammers an das Bayerische Staatsministeriums, Berlin, Juli 1936; ebd., Bl. 59: Hans Heinrich Lammers an das Staatsministerium des Innern [Bayerns], 14.3.1936; BA Berlin, R 43 II/1029a, Bl. 75: Schreiben zu Rk. 17430 B., 25.2.1938.

5 BA Berlin, R 43 II/1235a, Bl. 52: Der Kultusminister an den Chef der Präsidialkanzlei, Staatssekretär Meissner, 14.5.1935.

6 Kater 2006: 95. BA Berlin, NS 21/164, Bl. 1-4: Martin Rudolph, Bericht über Erforschung und Aufnahme der bäuerlichen Baukultur in der Gottschee, Forschungsstätte für germanisches Bauwesen, 3.12.1941.

7 Politisches Archiv des Auswärtigen Amtes (PAAA), Kult VI A, 2, Nr. 11, 6: Vortrag Bolko von Richthofen, Die Slawen in Schlesien, anlässlich der XVI. Tagung der Stiftung für Volks- und Kulturbodenforschung in Bad Salzbrunn vom 18.-20.10.1929, Bl. 506-508, hier Bl. 506.

8 Stellvertretend Horwath 1942; Knapp 1942; Schmid 1942; Storm 1941; Tintelnot 1941; Weinelt 1942.

9 Schweiz. Burgenarchiv, Akte „Ausstellung Burgen von Basel und Umgebung“: Bericht des Kantons-Chemikers Dr. R. Viollier an Carl Roth, 21.4.1932.

10 Bayerisches Hauptstaatsarchiv (Bay HStA), NL Esterer 28: Der geschäftsführende Vorsitzende des Pfälzischen Verkehrsverbands, Landrat Unckrich, an Rudolf Esterer, 30.6.1960.

11 Bay HStA, StK 7516: Georg Lill \& Rudolf Pfister, Niederschrift über die Sitzung wegen der Burgruine Trifels, 30.3.1937, S. 1.

12 Ebd., S. 4; BA Berlin, R 73/16338, Bl. 6 f.: Georg Lill an Paul Clemen und an den Stadtrat in Kaiserslautern, 9.7.1934; Landesarchiv Speyer (LASp), V 154, Nr. 4: Rudolf Esterer an Friedrich Sprater, 3.5.1937.

13 LASp, H 3, Nr. 1906 III, Bl. 25: Georg Lill an die Regierung der Pfalz in Speyer, 24.2.1937.

14 Ebd., Bl. 48-54, Bl. 50: Dossier Der Bayerische Ministerpräsident an die Kreisregierung der Pfalz in Speyer zur gefl. vertraulichen Kenntnisnahme, 6.4.1937.

15 Bay HStA, StK 7516: Georg Lill an Ludwig Siebert, 6.10.1937.

16 Historisches Museum der Pfalz in Speyer (HMP Speyer), Ortsakten Annweiler, Trifels: Friedrich Sprater, Bericht: Trifels. Ausgrabungen vom 16. bis 31.5.1937, datiert 4.6.1937, Bl. 1.

17 Archiv der Deutschen Burgenvereinigung e. V., Akte „Trifels. Ruine, b. Annweiler. Landau/Rheinpfalz": Burg Trifels. Ein Gutachten von Bodo Ebhardt, 28.11.1936, S. 10; LASp, H 3, Nr. 1906 III, Bl. 23 f.: Regierung der Pfalz an das Landesamt für Denkmalpflege in München, 3.2.1937. 
18 Ebd., Bl. 70 f.: Niederschrift über die Besprechung am 26.5.37 in Annweiler hinsichtlich der Arbeiten am Trifels, 31.5.1937.

19 Ebd., Bl. 152-156, hier Bl. 152 f.: Bodo Ebhardt, Vorschläge für eine Arbeitsanweisung des örtlichen Bauleiters bei den Ausgrabungen usw. auf dem Trifels, undatiert.

20 HMP Speyer, Ortsakten Annweiler, Trifels, Bl. 1: Friedrich Sprater, Bericht: Trifels. Ausgrabungen vom 5.-16.4.1937, datiert 18.4.1937; ebd., Friedrich Sprater, Bericht: Trifels. Ausgrabungen vom 17. bis 30.4.1937, datiert 2.5.1937.

21 Ebd., Regierung der Pfalz an den Bayerischen Ministerpräsidenten, 1.4.1937.

22 Bay HStA, StK 7518: Rudolf Esterer an Ludwig Siebert, 12.10.1937.

23 LASp, H 3, Nr. 1906 III, Bl. 199: Landbauamt Speyer an die Regierung der Pfalz, 20.9.1937.

24 Bay HStA, StK 7518: Ludwig Siebert an die Kreisregierung der Pfalz in Speyer, 23.10.1937; ebd.: Rudolf Esterer an Ludwig Siebert und das Staatsministerium für Unterricht und Kultus, 19.7.1938.

25 LASp, V 154, Nr. 4: Bodo Ebhardt an Friedrich Sprater, 9.6.1938.

26 HMP Speyer, Ortsakten Annweiler, Trifels, Bl. 1: Friedrich Sprater, Bericht: Trifels. Ergebnis der Ausgrabungen vom 15.7. bis 31.8.1937, datiert 1.9.1937.

27 Ebd., Bl. 1 f.: Friedrich Sprater, Trifels. Ergebnis der Ausgrabungen in der Zeit von Anfang April bis Ende November 1937, undatiert.

28 Erst 2001 erschien die vollständige mittelalterarchäologische und bauhistorische Auswertung der noch vorhandenen Dokumentation aus den 1930er Jahren, vgl. Meyer 2001.

29 LASp, H 3, Nr. 1906 III, Bl. 228: Rembert Ramsauer an die Regierung der Pfalz in Speyer, 24.1.1939.

30 LASp, V 154, Nr. 4, Bl. 1, 4-8: Inhaltsplan für des Trifelswerk (2. Fassung vom 1.6.1939), Hervorhebung im Original; LASp, H 3, Nr. 1906 III, Bl. 237-244: Dr. Zint, Saarpfälzisches Institut für Landes- und Volksforschung, an Oberregierungsrat Born in Speyer, 27.6.1939: Verzeichnis der verschiedenen Mitarbeiter in der Anlage und eine Niederschrift von Ernst Christmann zur Frage des Namens „Trifels“.

31 LASp, V 154, Nr. 4, Bl. 9: Inhaltsplan für das Trifelswerk (2. Fassung vom 1.6.1939), Hervorhebung im Original.

32 HMP Speyer, Akte „XG/M Historisches Museum Allgemein 1941-1942“: Ernst Christmann an Hermann Emrich, 17.12.1941.

33 HMP Speyer, Akte „X M. Postius. Hist. Museum IV, 1938“: Johannes Postius an Hermann Emrich, 14.1.1938; ebd.: Hermann Emrich an Johannes Postius, 4.11.1938.

34 HMP Speyer, Akte „XG/M Historisches Museum, Volksbildungsverband, Pfalz-Saar, V.D.A., Briefwechsel G. Dell, 1934-39“: Protokoll zur Versammlung der ordentlichen Mitglieder (Führerratsmitglieder) des Volksbildungsverbandes Saarpfalz e. V. am Freitag, 22.4.1938, nachmittags 17 Uhr im Rathaussaal zu Neustadt a. d. Weinstrasse, datiert 28.4.1938.

35 HMP Speyer, Akte „Trifels: Fotografien, ungeordnet, und sonstige Dokumente“: Arbeitsgemeinschaft der völkischen Wissenschaft im Kampfbund für deutsche Kultur in der Westmark (Historischer Verein der Pfalz) an das Bürgermeisteramt, 15.7.1933.

36 HMP Speyer, Akte „XG/M. Postius, Hist. Museum V, 1938“: Carl W. Scherer an Johannes Postius, 31.12.1938.

37 LASp, H 3, Nr. 1906 III, Bl. 283 f.: Rundschreiben Rembert Ramsauer an die Mitarbeiter, 24.11.1939; Bay HStA, StK 7516, Bl. 1 f.: Saarpfälzisches Institut für Landes- und Volksforschung, Dr. Hermann Emrich, an Ludwig Siebert, 24.3.1939.

38 Vgl. am Beispiel des Kantons Basel-Landschaft Staatsarchiv Baselland, NA 2080, Erziehung T4. 1, Kommission zur Erhaltung von Altertümern 1921-1928: Auszug aus dem Protokoll des Regierungsrates des Kantons Basel-Landschaft am 18.7.1933, datiert 22.7.1933.

39 Archiv der Gesellschaft Pro Vindonissa, Akte „Jg. 1938, Nr. 04.01.03“: Entwurf zu einem Aufruf (vermutlich von E. Finsterwald, Aargauische Kommission für Archäologischen Arbeitsdienst), undatiert; ebd., Nr 4: Vindonissa. Hasenberg, 1938-1935, Schweizerische Zentralstelle für Freiwilligen Arbeitsdienst, Geschäftsführender Ausschuss, Auszug aus dem Protokoll der Sitzung, 17.6.1936.

40 Staatsarchiv Kanton Basel-Stadt, Privat-Archive 88 K1, Akte „Archiv der Historischen und Antiquarischen Gesellschaft zu Basel. Zuschriften. Mitteilungen. Gesuche. Dankschreiben usw. Von Nichtmitgliedern 1841-1934-36“: Der Technische Arbeitsdienst Basel 
(Kantonales Arbeitsamt Basel) an die Historische und Antiquarische Gesellschaft zu Basel, 28.8.1934.

41 Fahlbusch 1999: 297-438; und 2002. PAAA, Kult VI A 2-FOG, 9, Nr. 5, Kult. A 1807 * 37: Niederschrift über die am 17. Sept. 1936 abgehaltene Besprechung in Oberkirch; ebd.,: Protokoll der Tagung der Westdeutschen Forschungsgemeinschaft am 12.-14. März 1937 in Worms; ebd., Kult VI A 2-FOG, Vol. 10 (11): Tagung der Historischen Arbeitsgemeinschaft am Oberrhein am 26. Sept. 1937 zu Basel.

42 Archiv der Gesellschaft Pro Vindonissa, Akte „Jg. 1933-1937“: Aufruf: „Freiwilliger Arbeitsdienst Vindonissa 1935“; ebd., Akte „Jg. 1927-1942, Nr. 05.05.01“: R. Laur-Belart, „Freiwilliger Arbeitsdienst Vindonissa 1936“, in: Badener Tagblatt, 11.11.1936; Schweiz. Burgenarchiv, Akte „Aktionskomitee für die Ausgrabungen auf Alt-Tierstein am Horn 1934-1936“, Bl. 1-6.

43 Ebd.: Ausgrabung Tierstein. IV. Rapport über die 4. Grabungswoche vom 25.-30. Juni 1934.

44. Zentralbibliothek Solothurn, NL TAT_E 3.2.55: Eugen Tatarinoff an Wilhelm Amrein, 8.2.1927.

45 Staatsarchiv Kanton Aargau, CH-000051-7 ZwA 1987.0013/0005: Archaeologischer Arbeitsdienst der Schweiz. Ein Werk des freiwilligen Arbeitsdienstes, undatiert [vermutlich 1936].

46 Schweiz. Burgenarchiv, Akte „Aktionskomitee für die Ausgrabungen auf Alt-Tierstein am Horn 1934-1936“: Ausgrabung Tierstein. IV. Rapport über die 4. Grabungswoche vom 25.-30. Juni 1934.

47 BA Berlin, R 73/16338, Bl. 4 f.: Paul Clemen, Kommission für die Denkmälerstatistik der Rheinprovinz, an die Notgemeinschaft der Deutschen Wissenschaft, 8.8.1934.

48 BA Berlin, R 74/15206: REM an die Deutsche Forschungsgemeinschaft, Walter Schulz, 27.4.1939; BA Berlin, R 73/16563, Bl. 11: Hans Reinerth an Deutsche Forschungsgemeinschaft, 14.3.1935; ebd., Bl. 12: Der Vorsitzende des Kreisausschusses des Landkreises Goslar an die Deutsche Forschungsgemeinschaft, 7.3.1935; ebd., Bl. 44: Der Landrat des Landkreises Goslar an die Notgemeinschaft der Deutschen Wissenschaft, 16.5.1934.

49 BA Berlin, NS 21/827: Forschungs- und Lehrgemeinschaft „Das Ahnenerbe“, Jahrestagungen, Bericht über die Kieler Tagung 1939.

50 PAAA Kult VI A, 2, Nr. 11, 6: Vortrag von Richthofen, Bl. 506.

\section{Literatur}

Ammann, Hektor 1935. Bericht des Ausgrabungsausschusses. Argovia (47, Sonderausgabe: Die Ausgrabungen im Fricktal) 1934/1935: 3-12.

Aubin, Hermann 1937. Zur Erforschung der deutschen Ostbewegung. Deutsches Archiv für Landes- und Volksforschung (1): 37-70, 309-331, 562-602.

Backes, Klaus 1988. Hitler und die bildenden Künste. Kulturverständnis und Kunstpolitik im Dritten Reich. Köln: DuMont.

Backes, Klaus 2000. „Der Führer wünscht eine große Jugendherberge am Trifels“. Adolf Hitlers Rolle als Mäzen beim Teil-Aufbau der Reichsburg. Heimat-Jahrbuch des Landkreises Südliche Weinstraße (22): 117-121.

Bentz, Emma 2008. „More than a Village“: On the Medieval Countryside as an Archaeological Field of Study. In: Nathan Schlanger und Jarl Nordbladt (Hg.). Archives, Ancestors, Practices: Archaeology in the Light of its History. New York: Berghahn Books: 97-107.

Biller, Thomas 1998. Die Adelsburg in Deutschland. Entstehung, Gestalt, Bedeutung. München: Deutscher Kunstverlag.

Biller, Thomas und G. Ulrich Grossmann 2002. Burg und Schloss. Der Adelssitz im deutschsprachigen Raum. Regensburg: Schnell und Steiner.

Böhme, Horst Wolfgang, Reinhard Friedrich und Barbara Schock-Werner (Hg.) 2004. Wörterbuch der Burgen, Schlösser und Festungen. Stuttgart: Reclam. 
Brackmann, Albert 1937. Die Ausgrabungen in Zantoch. Deutsches Archiv für Landes- und Volksforschung (1): 174-179.

Brandi, Karl 1935. Die Ausgrabung der Pfalz Werla durch Regierungs-Baurat Dr. K. Becker. Berlin: Weidmann (= Nachrichten von der Gesellschaft der Wissenschaften zu Göttingen, Philologisch-Historische Klasse, Fachgruppe II, Mittlere und Neuere Geschichte, Neue Folge 1,2).

Castellani Zahir, Elisabeth 1997. Echt falsch und doch schön alt. Die Wiederherstellung der Hohkönigsburg im Elsass 1900 bis 1908. Zeitschrift für Schweizerische Archäologie und Kunstgeschichte (54): 141-152.

Deutsche Burgenvereinigung e. V. (Hg.) 1999. Burgen in Mitteleuropa. Ein Handbuch. 2 Bde. Stuttgart: Theiss.

Die Denkmalpflege/Deutsche Kunst und Denkmalpflege (ab 1934) 1899-1935.

Dilly, Heinrich 1979. Kunstgeschichte als Institution. Studien zur Geschichte einer Disziplin. Frankfurt am Main: Suhrkamp.

Dolff-Bonekämper, Gabi 2004. Wahr oder falsch. Denkmalpflege als Medium nationaler Identitätskonstruktionen. In: Otto Gerhard Oexle, Áron Petneki und Leszek Zygner (Hg.). Bilder gedeuteter Geschichte. Das Mittelalter in der Kunst und Architektur der Moderne. 2. Teilbd. Göttingen: Wallstein: 231-285.

Dubler, Reto et al. 2006. Vom Dübelstein zur Waldmannsburg. Adelssitz, Gedächtnisort und Forschungsprojekt. Basel: Schweizerischer Burgenverein (= Schweizer Beiträge zur Kulturgeschichte und Archäologie des Mittelalters, 33).

Eberhardt, Gisela 2008. Methodological Reflections on the History of Excavation Techniques. In: Nathan Schlanger und Jarl Nordbladt (Hg.). Archives, Ancestors, Practices: Archaeology in the Light of its History. New York: Berghahn Books: 89-96.

Ebhardt, Bodo 1898-1908: Deutsche Burgen. 9 Bde. Berlin: Ernst Wasmuth.

Ebhardt, Bodo 1938. Burg Trifels. Untersuchungen zur Baugeschichte. Braubach: Burgverlag.

Erb, Hans 1935. Ausgrabung Tierstein 1934. Bericht des Ausgrabungsleiters Hans Erb. Argovia (47): 13-94.

Fahlbusch, Michael 1999. Wissenschaft im Dienst der nationalsozialistischen Politik? Die "Volksdeutschen Forschungsgemeinschaften" von 1931-1945. Baden-Baden: Nomos.

Fahlbusch, Michael 2000. Für Volk, Führer und Reich! Die Volksdeutschen Forschungsgemeinschaften und Volkstumspolitik, 1931-1945. In: Doris Kaufmann (Hg.). Geschichte der KaiserWilhelm-Gesellschaft im Nationalsozialismus. Bestandsaufnahme und Perspektiven der Forschung. 2 Bde. Göttingen: Wallstein: 468-489.

Fahlbusch, Michael 2002. Zwischen Kollaboration und Widerstand. Zur Tätigkeit schweizerischer Kulturwissenschaftler in der Region Basel während des Dritten Reichs. Basler Zeitschrift für Geschichte und Altertumskunde (102): 47-74.

Fahlbusch, Michael 2008. Hektor Ammann. In: Ingo Haar und Michael Fahlbusch (Hg.). Handbuch der völkischen Wissenschaften. Personen - Institutionen - ForschungsprogrammeStiftungen. München: Saur: 38-43.

Falser, Michael S. 2008. Zwischen Identität und Authentizität. Zur politischen Geschichte der Denkmalpflege in Deutschland. Dresden: Thelem.

Fehring, Günter P. 2000. Die Archäologie des Mittelalters. Eine Einführung. 3. Aufl. Darmstadt: WBG/Theiss.

Fischer, Ludger 2010. Bodo Ebhardt. Versuche baukünstlerischer Denkmalpflege. Restaurierungen, Rekonstruktionen und Neubauten von Burgen, Schlössern und Herrenhäusern von 1899 bis 1935. Braubach: Deutsche Burgenvereinigung.

Fleck, Ludwik 1980 [1935]. Entstehung und Entwicklung einer wissenschaftlichen Tatsache. Einführung in die Lehre vom Denkstil und Denkkollektiv. Frankfurt am Main: Suhrkamp.

Fleck, Ludwik 1983. Erfahrung und Tatsache. Gesammelte Aufsätze. Frankfurt am Main: Suhrkamp.

Fleischner, Susanne 1999. „Schöpferische Denkmalpflege“. Kulturideologie des Nationalsozialismus und Positionen der Denkmalpfleger. Münster: LIT.

Freund, Wolfgang 2006. Volk, Reich und Westgrenze. Deutschtumswissenschaften und Politik in der Pfalz, im Saarland und im annektierten Lothringen 1925-1945. Saarbrücken: SDV.

Galison, Peter 1996. Computer Simulations and the Trading Zone. In: ders. und David J. Strump (Hg.). The Disunity of Science: Boundaries, Contexts, and Power. Stanford: Stanford University Press: 118-157. 
Geuter, Ulfried 1984. Die Professionalisierung der deutschen Psychologie im Nationalsozialismus. Frankfurt am Main: Suhrkamp.

Glatz, Joachim 2005. Rudolf Esterer und die Schöpferische Denkmalpflege. Der Ausbau des Trifels. In: Ingrid Scheurmann (Hg.). Zeitschichten. Erkennen und erhalten - Denkmalpflege in Deutschland. München: Deutscher Kunstverlag: 142-145.

Grabolle, Roman, Uwe Hoßfeld und Klaus Schmidt 2003. Ur- und Frühgeschichte in Jena 19301945: Lehren, Forschen und Graben für Germanien? In: Uwe Hoßfeld, Jürgen John, Oliver Lemuth und Rüdiger Stutz (Hg.). „Kämpferische Wissenschaft“. Studien zur Universität Jena im Nationalsozialismus. Köln: Böhlau Verlag: 868-912.

Grunwald, Susanne 2009. Potentiale der Burgwallforschung. Sächsische Archäologie in der ersten Hälfte des 20. Jahrhunderts. In: Judith Schachtmann, Michael Strobel und Thomas Widera (Hg.). Politik und Wissenschaft in der prähistorischen Archäologie. Perspektiven aus Sachsen, Böhmen und Schlesien. Göttingen: Vandenhoeck \& Ruprecht: 149-168.

Grunwald, Susanne 2014. „Das ergab aber ein so buntes und wenig eindrucksvolles Bild“. Zu den Anfängen der archäologischen Kartographie in Deutschland (1870-1914). EAZ (53/1): 5-34.

Grünert, Heinz 2002. Gustaf Kossinna - ein Wegbereiter der nationalsozialistischen Archäologie. In: Achim Leube und Morten Hegewisch (Hg.). Prähistorie und Nationalsozialismus. Die mittel- und osteuropäische Ur- und Frühgeschichtsforschung in den Jahren 1933-1945. Heidelberg: Synchron: 307-320.

Hachtmann, Rüdiger 2007. Wissenschaftsmanagement im „Dritten Reich“. Geschichte der Generalverwaltung der Kaiser-Wilhelm-Gesellschaft. 2 Bde. Göttingen: Wallstein.

Hagner, Michael 1997. Zwei Anmerkungen zur Repräsentation in der Wissenschaftsgeschichte. In: Hans-Jörg Rheinberger, ders. und Bettina Wahrig-Schmidt (Hg.). Räume des Wissens. Repräsentation, Codierung, Spur. Berlin: Akademie Verlag: 339-354.

Halle, Uta 2008. Ur- und Frühgeschichte. In: Jürgen Elvert und Jürgen Nielsen-Sikora (Hg.). Kulturwissenschaften und Nationalsozialismus. Stuttgart: Franz Steiner: 109-166.

Hausmann, Frank-Rutger 2001. „Termitenwahn“ - Die Bedeutung der Gemeinschaftsforschung für die NS-Wissenschaft. In: Georg Bollenbeck und Clemens Knobloch (Hg.). Semantischer Umbau der Geisteswissenschaften nach 1933 und 1945. Heidelberg: Winter: 58-79.

Heftrig, Ruth, Olaf Peters und Barbara Maria Schellewald (Hg.) 2008. Kunstgeschichte im „Dritten Reich". Theorien, Methoden, Praktiken. Berlin: Akademie Verlag.

Hinz, Hermann 1981. Motte und Donjon. Zur Frühgeschichte der mittelalterlichen Adelsburg. Köln: Rheinland Verlag (= Zeitschrift für Archäologie des Mittelalters, Beiheft 1).

Horwath, Walter 1942. Die Burgen des Deutschen Ordens in Preußen. Deutsches Archiv für Landes- und Volksforschung (6): 74-96.

Hotz, Walter 1937. Staufische Reichsburgen am Mittelrhein. Berlin: Deutscher Kunstverlag.

Hotz, Walter 1940. Kaiserpfalzen und Ritterburgen in Franken und Thüringen. Berlin: Deutscher Kunstverlag.

Jacobeit, Wolfgang, Hannjost Lixfeld und Olaf Bockhorn (Hg.) 1994. Völkische Wissenschaft. Gestalten und Tendenzen der deutschen und österreichischen Volkskunde in der ersten Hälfte des 20. Jahrhunderts. Wien: Böhlau Verlag.

Jankuhn, Herbert 1943. Die Ausgrabungen in Haithabu (1937-1939). Vorläufiger Grabungsbericht. Berlin-Dahlem: Ahnenerbe-Stiftung Verlag.

Jungert, Michael 2010. Was zwischen wem und warum eigentlich? Grundsätzliche Fragen der Interdisziplinarität. In: ders., Elsa Romfeld, Thomas Sukopp und Uwe Voigt (Hg.). Interdisziplinarität. Theorie, Praxis, Probleme. Darmstadt: WBG: 1-12.

Kater, Michael H. 2006. Das „Ahnenerbe“ der SS 1935-1945. Ein Beitrag zur Kulturpolitik des Dritten Reiches. 4. Aufl. München: R. Oldenbourg Verlag.

Knapp, Werner 1942. Der Burgentypus in der Steiermark. Weg und Ziel neuzeitlicher Burgenforschung. Deutsches Archiv für Landes- und Volksforschung (6): 867-879.

Kocka, Jürgen 1987. Einleitung. In: ders. (Hg.). Interdisziplinarität. Praxis - Herausforderung Ideologie. Frankfurt am Main: Suhrkamp: 7-14.

Kohler, Robert E. 2002. Landscapes and Labscapes: Exploring the Lab-Field Border in Biology. Chicago: University of Chicago Press.

Krämer, Werner 2002. Gerhard Bersu, ein deutscher Prähistoriker 1889-1964. Mainz: Zabern.

Latour, Bruno 2006: Drawing Things Together: Die Macht der unveränderlichen mobilen Elemente. In: Andréa Belliger und David J. Krieger (Hg.). ANThology. Ein einführendes Handbuch zur Akteur-Netzwerk-Theorie. Bielefeld: transcript: 259-307. 
Laur-Belart, Rudolf 1943. Das neue Institut für Ur- und Frühgeschichte der Schweiz. Ur-Schweiz (7/1): $1-8$.

Lenoir, Timothy 1997. Instituting Science: The Cultural Production of Scientific Disciplines. Stanford: Stanford University Press.

Leube, Achim und Morten Hegewisch (Hg.) 2002. Prähistorie und Nationalsozialismus. Die mittel- und osteuropäische Ur- und Frühgeschichtsforschung in den Jahren 1933-1945. Heidelberg: Synchron.

Mercer, David 2006. The Trouble with Paradigms: A Historiographical Study on the Development of Ideas in the Discipline of Castle Studies. Archaeological Dialogues (13/1): 93-109.

Merz, Walther 1909-1914: Die Burgen des Sisgaus. 4 Bde. Aarau: H. R. Sauerländer.

Meyer, Bernhard 2001. Burg Trifels. Die mittelalterliche Baugeschichte. Kaiserslautern: Institut für Pfälzische Geschichte und Volkskunde.

Motschi, Andreas 2000. Eine Pioniergrabung auf Schloss Hallwyl (1910-1916). Mittelalter (5/3): 84-90.

Neumann, Gotthard 1929. Die Entwicklung der Aunjetitzer Keramik in Mitteldeutschland. Prähistorische Zeitschrift 20 (1-2): 70-144.

Neumann, Gotthard 1940. Kyffhäuserstudien I. Die vorgeschichtliche Besiedlung des Baugrundes der Reichsburg Kyffhausen (Unter- und Oberburg). Zeitschrift des Vereins für Thüringische Geschichte 34: 318-371.

Oberkrome, Willi 1991. Reformansätze in der deutschen Geschichtswissenschaft der Zwischenkriegszeit. In: Michael Prinz und Rainer Zitelmann (Hg.). Nationalsozialismus und Modernisierung. Darmstadt: WBG: 216-238.

Oberkrome, Willi 1993. Volksgeschichte Methodische Innovation und völkische Ideologisierung in der deutschen Geschichtswissenschaft 1918-1945. Vandenhoeck \& Ruprecht, Göttingen

Oexle, Otto Gerhard 1996. Geschichtswissenschaft im Zeichen des Historismus Studien zur Problemgeschichte der Moderne. Vandenhoeck \& Ruprecht, Göttingen.

Oexle, Otto Gerhard 2004. Bilder gedeuteter Geschichte. Eine Einführung. In: ders., Áron Petneki und Leszek Zygner (Hg.). Bilder gedeuteter Geschichte. Das Mittelalter in der Kunst und Architektur der Moderne. 2. Teilbd. Göttingen: Wallstein: 11-30.

Pape, Wolfgang 2002. Zur Entwicklung des Faches Ur- und Frühgeschichte in Deutschland bis 1945. In: Achim Leube und Morten Hegewisch (Hg.). Prähistorie und Nationalsozialismus. Die mittel- und osteuropäische Ur- und Frühgeschichtsforschung in den Jahren 1933-1945. Heidelberg: Synchron: 163-226.

Patel, Kiran Klaus 2003. „Soldaten der Arbeit“: Arbeitsdienste in Deutschland und den USA 19331945. Göttingen: Vandenhoeck \& Ruprecht.

Piper, Otto 1912. Burgenkunde. Bauwesen und Geschichte der Burgen zunächst innerhalb des Deutschen Sprachgebietes. 3. Aufl. München: Verlag R. Piper.

Pütz, Frank 2002. Die Burg im Nationalsozialismus. Burgenrezeption in der deutschen Architektur zwischen 1933 und 1945. In: Heiko Laß (Hg.). Mythos, Metapher, Motiv. Untersuchungen zum Bild der Burg seit 1500. Alfeld/Leine: Coppi: 43-66.

Reinhardt, Carsten 2001. Disciplines, Research Fields, and their Boundaries. In: ders. (Hg.). Chemical Sciences in the $20^{\text {th }}$ Century: Bridging Boundaries. Weinheim: Wiley-CH: 1-13.

Reinhardt, Carsten 2006. Shifting and Rearranging: Physical Methods and the Transformation of Modern Chemistry. Sagamore Beach, MA: Science History Publications.

Schachtmann, Judith, Michael Strobel und Thomas Widera (Hg.). Politik und Wissenschaft in der prähistorischen Archäologie. Perspektiven aus Sachsen, Böhmen und Schlesien. Göttingen: Vandenhoeck \& Ruprecht.

Schöbel, Gunter 2002. Hans Reinerth. Forscher - NS-Funktionär - Museumsleiter. In: Achim Leube und Morten Hegewisch (Hg.). Prähistorie und Nationalsozialismus. Die mittel- und osteuropäische Ur- und Frühgeschichtsforschung in den Jahren 1933-1945. Heidelberg: Synchron: 321-396.

Schmid, Bernhard 1942. Die Burgen des Deutschen Ostens in Preußen. Deutsches Archiv für Landes- und Volksforschung (6): 74-96.

Schmoll, Friedemann 2009. Die Vermessung der Kultur Der „Atlas der deutschen Volkskunde“ und die Deutsche Forschungsgemeinschaft 1928-1980. Franz Steiner, Stuttgart.

Schreiner, Klaus 1985. Führertum, Rasse, Reich. Wissenschaft von der Geschichte nach der nationalsozialistischen Machtergreifung. In: Peter Lundgreen (Hg.): Wissenschaft im Dritten Reich. Frankfurt am Main: Suhrkamp: 163-252. 
Schuchhardt, Carl 1931. Die Burg im Wandel der Weltgeschichte. Potsdam: Akademische Verlagsgesellschaft Athenaion.

Siebert, Ludwig 1941: Deutsches Kulturschaffen als völkische Pflicht. In: ders. (Hg.). Wiedererstandene Baudenkmale. Ausgewählte Arbeiten aus dem Ludwig-Siebert-Programm zur Erhaltung bayerischer Baudenkmale. München: Selbstverlag: 7-10.

Simon, Christian 1995. Hektor Ammann - Neutralität, Germanophilie und Geschichte. In: Aram Mattioli (Hg.). Intellektuelle von rechts: Ideologie und Politik in der Schweiz 1918-1939. Zürich: Orell Füssli: 29-53.

Sprater, Friedrich 1937. Der Trifels. Ein Denkmal deutscher Macht im ersten Reich. GermanenErbe (2/6): 178-186.

Sprater, Friedrich 1945. Der Trifels. Die deutsche Gralsburg. Speyer: Verlag des Historischen Museums der Pfalz.

Stein, Günter 1975. Trifels und Hohkönigsburg. Zitate und Gedanken zum Wiederaufbau zweier Burgruinen. In: Alfons Schäfer (Hg.). Festschrift für Günther Haselier. Karlsruhe: Thorbecke: 373-404.

Steuer, Heiko 1997/1998. Entstehung und Entwicklung der Archäologie des Mittelalters und der Neuzeit in Mitteleuropa - Auf dem Weg zu einer eigenständigen Mittelalterkunde. Zeitschrift für die Archäologie des Mittelalters und der Neuzeit (25/26): 19-38.

Storm, Carl 1941. Zur deutschen Burgenforschung. Bemerkungen von seiten der Burgengeographie. Deutsches Archiv für Landes- und Volksforschung (5): 118-142.

Szöllösi-Janze, Margit 2000. Der Wissenschaftler als Experte. Kooperationsverhältnisse von Staat, Militär, Wirtschaft und Wissenschaft, 1914-1933. In: Doris Kaufmann (Hg.). Geschichte der Kaiser-Wilhelm-Gesellschaft im Nationalsozialismus. Bestandsaufnahme und Perspektiven der Forschung: 46-64.

Teibenbacher, Peter 2003. Die Nation als Burg. Burgen-Romantik und Burgen-Ideologie im Nationalsozialismus. In: Helmut Bräuer, Gerhard Jaritz und Käthe Sonnleitner (Hg.). Viatori per urbes castraque. Festschrift für Herwig Ebner zum 75. Geburtstag. Graz: Institut für Geschichte der Karl-Franzens-Universität: 685-691.

Tintelnot, Hans 1941. Die Stellung der schlesischen Baukunst in der ostdeutschen Architektur des Mittelalters. Deutsches Archiv für Landes- und Volksforschung (5): 591-602.

Wallraff, Horst 2008. Regional- und Landesgeschichte. In: Jürgen Elvert und Jürgen Nielsen-Sikora (Hg.). Kulturwissenschaften und Nationalsozialismus. Stuttgart: Franz Steiner: 246-288.

Weinelt, Herbert 1942. Der mittelalterliche Wehrbau einer ostmitteldeutschen Rodungslandschaft. Die Burgen des Kreises Freiwaldau im Ostsudetenland. Deutsches Archiv für Landesund Volksforschung (6): 148-168.

Weingart, Peter, Martin Carrier und Wolfgang Krohn 2007. Nachrichten aus der Wissensgesellschaft. Analysen zur Veränderung der Wissenschaft. Weilerswist: Velbrück.

Wolnik, Gordon 2004. Mittelalter und NS-Propaganda. Mittelalterbilder in den Print-, Ton- und Bildmedien des Dritten Reiches. Münster: LIT Verlag.

Fabian Link

Johann Wolfgang Goethe-Universität Frankfurt

am Main

Grüneburgplatz 1

D-60629 Frankfurt am Main

Germany

E-Mail: f.link@em.uni-frankfurt.de 